\title{
Correspondence
}

\section{Hybrid Bit-to-Symbol Mapping for Spatial Modulation}

2 Ping Yang, Yue Xiao, Lu Yin, Qian Tang,

3 Shaoqian Li, Senior Member, IEEE, and Lajos Hanzo, Fellow, IEEE

4 Abstract-In spatial modulation (SM), the information bit stream is di5 vided into two different sets: the transmit antenna index bits (TA-bits) and 6 the amplitude and phase modulation bits (APM-bits). However, the con7 ventional bit-to-symbol mapping (BTS-MAP) scheme maps the APM-bits 8 and the TA-bits independently. For exploiting their joint benefits, we 9 propose a new BTS-MAP rule based on the traditional 2-D Gray mapping 10 rule, which increases the Hamming distance (HD) between the symbol 11 pairs detected from the same transmit antenna (TA) and simultaneously 12 reduces the average HD between the symbol pairs gleaned from different

13 TAs. Based on the analysis of the distribution of minimum Euclidean 14 distance (MED) of SM constellations, we propose a criterion for the con15 struction of a meritorious BTS-MAP for a specific SM setup, with no need 16 for additional feedback links or extra computational complexity. Finally, 17 Monte Carlo simulations are conducted for confirming the accuracy of our 18 analysis.

19 Index Terms-Gray mapping, hamming distance (HD), multiple-input20 multiple-output (MIMO), spatial modulation (SM).

\section{INTRODUCTION}

22 Spatial modulation (SM) is a new 3-D hybrid modulation scheme 23 conceived for multiple-input-multiple-output (MIMO) transmission, 24 which exploits the indexes of the transmit antennas (TAs) as an 25 additional dimension invoked for transmitting information, apart from 26 the classic 2-D amplitude and phase modulation (APM) [1]-[5]. In $27 \mathrm{SM}$, the information bit stream is divided into two different sets: the 28 bits transmitted through the TA indexes and the APM constellations 29 [6]. For simplicity, we refer to these two sets of bits as TA-bits and 30 APM-bits.

31 In conventional 2-D APM constellations, the choice of the bit-to32 symbol mapping (BTS-MAP) rule plays an important role in determin33 ing the achievable bit error ratio (BER) performance [7]. For example, 34 an optimized BTS-MAP is capable of providing a low error floor in 35 both bit-interleaved coded modulation and in its iterative decoding and

Manuscript received July 13, 2014; revised April 23, 2015; accepted July 2, 2015. This work was supported in part by the European Research Council's Advanced Fellow Grant, by the National Science Foundation of China under Grant 61471090 , by the National High-Tech R\&D Program of China (863 Project) under Grant 2014AA01A707, and by the Open Research Fund of the National Mobile Communications Research Laboratory of Southeast University under Grant 2013D05. The review of this paper was coordinated by Dr. C. Yuen.

P. Yang, L. Yin, Q. Tang, and S. Li are with the National Key Laboratory of Science and Technology on Communications, University of Electronic Science and Technology of China, Sichuan 611731, China (e-mail: yplxw@163.com; 759562945@qq.com; qian.tang@163.com; 1sq@uestc.edu.cn).

Y. Xiao is with the National Key Laboratory of Science and Technology on Communications, University of Electronic Science and Technology of China, Sichuan 611731, China, and also with National Mobile Communications Research Laboratory, Southeast University, Nanjing 210096, China (e-mail: xiaoyue@uestc.edu.cn).

L. Hanzo is with the School of Electronics and Computer Science, University of Southampton, Southampton SO17 1BJ, U.K. (e-mail: 1h@ecs.soton.ac.uk).

Color versions of one or more of the figures in this paper are available online at http://ieeexplore.ieee.org.

Digital Object Identifier 10.1109/TVT.2015.2453216 demodulation aided counterpart (BICM-ID) [8]. It is widely known 36 that, for equally likely and statistically independent 2-D APM constel- 37 lations, Gray mapping is optimal in terms of minimizing the BER [7]. 38 In general, the optimal BTS-MAP depends on the specific geometry 39 of the signal constellation, particularly on the location of the phasors 40 separated by the minimum Euclidean distance (MED) [9]. Compared 41 with APM schemes, SM has a higher constellation dimension and a 42 different distribution of MED due to its hybrid modulation principle. 43 Hence, the classic Gray mapping proposed for 2-D constellations will 44 no longer achieve the optimal BER in fading MIMO channels.

Recently, the wide-ranging studies disseminated in [1], [4], [6], 46 and [10]-[14] have characterized some of the fundamental properties 47 of SM, such as its energy efficiency [12], [13] and the effects of 48 power imbalance [14]. In these contributions, a general framework was 49 established for the BTS-MAP rule of SM [1], where the APM-bits are 50 mapped according to the classic Gray mapping rule, whereas the TA- 51 bits are mapped to the active TA index. Due to its intuitive nature 52 and low complexity, this rule has been considered in diverse SM- 53 based systems [15]-[18]. For example, in [15] and [16], this general 54 framework was developed for an arbitrary number of TAs and hence 55 strikes a flexible tradeoff in terms of the attainable BER performance 56 and capacity. In [17] and [18], this philosophy has been extended to 57 trellis coded modulation aided SM systems for the sake of achieving 58 reliable digital transmission. However, this classic BTS-MAP scheme 59 maps the APM-bits and the TA-bits to symbols independently and 60 hence may sacrifice the classic Gray-coded benefits. Moreover, the 61 MED distribution of SM was not considered in the design process of 62 the conventional BTS-MAP scheme, which is a measure of separation 63 between two constellation points, and as a result, it has a dominant 64 influence on the BER.

Against this background, the novel contributions of this treatise are 66 as follows.

- We propose a new BTS-MAP rule based on the classic Gray- 69 coded principles for exploiting the interaction of the TA-bits 70 and the APM-bits in fading channels, which differs from the 71 conventional BTS-MAP, since it reduces the average Hamming 72 distance (HD) between the SM symbol pair of the different TAs, 73 and simultaneously, it increases the HD between the symbol pair 74 of the same TA.

- Based on the analysis of the distribution of the MED, we propose 76 a criterion for the construction of a beneficial BTS-MAP rule 77 for a specific SM-MIMO setup, which is achieved without the 78 need for an additional feedback link and with no extra compu- 79 tational complexity. Finally, performance comparisons with the 80 conventional BTS-MAP scheme of [1] are provided for different 81 constellation sizes and signal-to-noise ratios (SNRs).

Notation: $(\cdot)^{*},(\cdot)^{\mathrm{T}}$, and $(\cdot)^{\mathrm{H}}$ denote conjugate, transpose, and 83 Hermitian transpose, respectively. The probability of an event is rep- 84 resented by $P(\cdot)$. Furthermore, $\|\cdot\|$ and $|\cdot|$ denote the Euclidean 85 norm and magnitude operators, respectively; whereas $\varepsilon(\mathbf{x}, \mathbf{y})$ is the 86 HD between the binary strings $\mathbf{x}$ and $\mathbf{y} . N_{t}$ is the number of TAs, 87 and $M$ is the size of the APM constellation adopted. Let $\mathbf{b}_{i}^{m}$ be 88 the transmit bit vector mapped to the SM symbol $\mathbf{x}_{i}$ and the APM 89 symbol $s_{i}^{m}$, which corresponds to TA $i$, whereas $\mathbf{b}_{j}^{k}$ is the transmit 90 bit vector mapped to the $\mathrm{SM}$ symbol $\mathbf{x}_{j}$ related to TA $j$ and the APM 91 


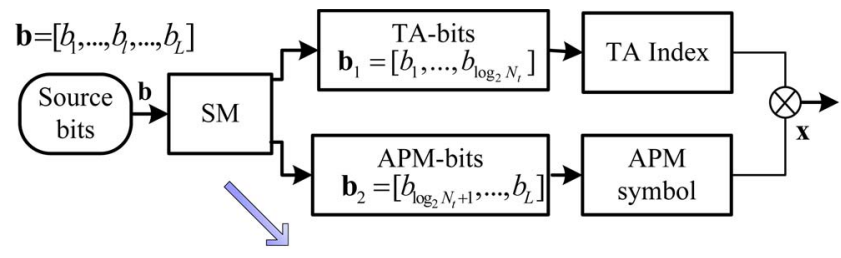

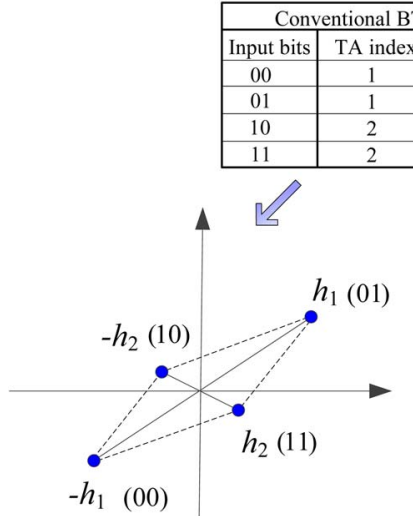

(a) $\min \left\{2\left|h_{1}\right|, 2\left|h_{2}\right|\right\}<\min \left\{\left|h_{1}-h_{2}\right|,\left|h_{1}+h_{2}\right|\right\}$

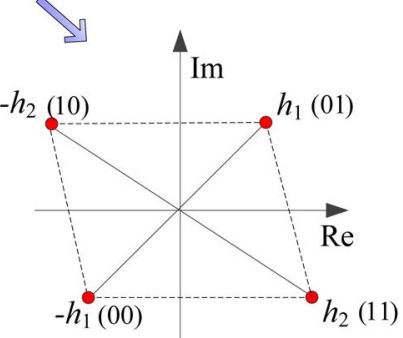

(b) $\min \left\{2\left|h_{1}\right|, 2\left|h_{2}\right|\right\} \geq \min \left\{\left|h_{1}-h_{2}\right|,\left|h_{1}+h_{2}\right|\right\}$
Fig. 1. Conventional BTS-MAP of SM: an example for the BPSK-modulated $(2 \times 1)$-element SM. (a) MED encountered on the same TA, where the conventional BTS-MAP is optimal. (b) MED encountered on different TAs, where the conventional BTS-MAP is suboptimal.

92 symbol $s_{j}^{k}$. The average HD between the symbol pair gleaned from 93 different TAs, termed as HDD, is defined as HDD $=\left(1 /\left(N_{t}\left(N_{t}-\right.\right.\right.$ 94 1) $\left.\left.M^{2}\right)\right) \sum_{i \neq j, i, j \in\left\{1, \ldots, N_{t}\right\}} \varepsilon\left(\mathbf{b}_{i}^{m}, \mathbf{b}_{j}^{k}\right)$. Moreover, the average HD 95 between the symbol pair detected from the same TA, termed as HDS, 96 is defined as HDS $=\left(1 /\left(N_{t} M(M-1)\right)\right) \sum_{i \in\left\{1, \ldots, N_{t}\right\}} \varepsilon\left(\mathbf{b}_{i}^{m}, \mathbf{b}_{i}^{k}\right)$. 97 For a fixed MIMO channel $\mathbf{H}, d_{\min }^{\mathrm{Same}}(\mathbf{H})$ is the MED between the 98 symbol pair of the same TA, $d_{\min }^{\mathrm{Diff}}(\mathbf{H})$ is the MED between the 99 symbol pair of different TAs, and the overall MED of a specific SM 100 is $d_{\min }=\min \left\{d_{\min }^{\mathrm{Same}}(\mathbf{H}), d_{\min }^{\text {Diff }}(\mathbf{H})\right\}$.

\section{II. Conventional Bit-To-Symbol MapPing Rule For 102 SPATIAL MODULATION}

103 Consider a MIMO system having $N_{t}$ transmit and $N_{r}$ receive 104 antennas. The $\left(N_{r} \times N_{t}\right)$-element channel matrix $\mathbf{H}$ is used for mod105 eling a flat-fading channel with elements having complex Gaussian 106 distributions with unit variance. We not only focus our attention on 107 the independent and identically distributed Rayleigh case but discuss 108 Nakagami- $m$ channels as well. Let $\mathbf{b}=\left[b_{1}, \ldots, b_{L}\right]$ be the transmit 109 bit vector in each time slot, which contains $L=\log _{2}\left(N_{t} M\right)$ bits. As 110 shown in Fig. 1, the input vector $\mathbf{b}$ is divided into two subvectors 111 of $\log _{2}\left(N_{t}\right)$ and $\log _{2}(M)$ bits, which are denoted by $\mathbf{b}_{1}$ and $\mathbf{b}_{2}$, 112 respectively. The bits in the subvector $\mathbf{b}_{1}$ are used for selecting a 113 unique TA index $q$ for activation, whereas the bits in the subvector $114 \mathbf{b}_{2}$ are mapped to a Gray-coded APM symbol $s_{l}^{q}$. Hence, the resultant $115 \mathrm{SM}$ symbol $\mathrm{x} \in \mathbb{C}^{N_{t} \times 1}$ is formulated as [6]

$$
\mathbf{x}=s_{l}^{q} \mathbf{e}_{q}
$$

116 where $\mathbf{e}_{q}\left(1 \leq q \leq N_{t}\right)$ is selected from the $N_{t}$-dimensional standard 117 basis vectors.

118 To expound a little further, we exemplify the binary phase-shift 119 keying (BPSK)-modulated $(2 \times 1)$-element SM in Fig. 1. As shown 120 in Fig. 1, $L=2$ input bits are divided into two single-bit streams, and 121 then, the first bit determines the activated TA (1 or 2), whereas the 122 second single bit generates the classic BPSK symbol $(+1$ or -1$)$. The aforementioned BTS-MAP method considers the APM-bits and the 123 TA-bits independently and hence facilitates a simple implementation 124 of SM.

However, this BTS-MAP method may result in a Gray-coding 126 penalty [9], which degrades the BER. Specifically, for the example 127 of BPSK-modulated $(2 \times 1)$-element SM in Fig. 1, assuming that the 128 channel matrix is $\mathbf{H}=\left[h_{1}, h_{2}\right]$, there are four received constellation 129 points denoted by $h_{1}, h_{2},-h_{1}$, and $-h_{2}$; and we investigate two 130 scenarios: 1) the MED is $d_{\min }=2\left|h_{2}\right|$; and 2) the MED is $d_{\min }=131$ $\min \left\{\left|h_{1}-h_{2}\right|,\left|h_{1}+h_{2}\right|\right\}$. Note that scenario (1) corresponds to the 132 case when the MED is encountered on the same TA (TA 2), whereas 133 scenario (2) corresponds to the case when the MED is encountered 134 on different TAs (between TAs 1 and 2). For scenario (1), the most 135 likely erroneously detected pattern is given by the nearest constellation 136 points $\left(-h_{2}, h_{2}\right)$. If the conventional BTS-MAP is adopted, these 137 points differ only in a single bit, i.e., by the difference between the 138 bits "10" and "11" in Fig. 1(a), whereas other adjacent constellation 139 points may differ in more than one bits. This mapping rule obeys 140 the concept of Gray mapping, where the probability of having a 141 bit error is minimized; hence, it has an optimal performance in this 142 specific scenario. However, for scenario (2), it is suboptimal, because 143 the nearest constellation points become $\left(-h_{1}, h_{2}\right)$, which differ in 144 two bits. This implies that more than one bit errors are associated 145 with the most likely error pattern of $\left(-h_{1}, h_{2}\right)$; hence, a performance 146 penalty will occur. This indicates that the MED distribution should be 147 considered in the design of BTS-MAP.

\section{Proposed BIT-To-SyMBOL MAPPING FOR SPATIAL MODULATION}

\section{A. Principle of the Proposed BTS-MAP}

We found in Section II that the design of the BTS-MAP scheme 152 depends on the MED, which may be achieved between the symbol 153 pair gleaned from the same TA or between the symbol pair from 154 different TAs. The conventional BTS-MAP rule does not consider this 155 distribution of the MED, and hence, it becomes suboptimal for some 156 channel scenarios, as illustrated in Fig. 1(b).

For exploiting the mapping gain of SM, we propose a new 158 BTS-MAP scheme based on traditional Gray-coded modulation. 159 To be specific, similar to the conventional BTS-MAP of SM, 160 the $L$-bit input vector $\mathbf{b}=\left[b_{1}, \ldots, b_{L}\right]$ is divided into a pair 161 of subvectors $\mathbf{d}=\left[d_{1}, \ldots, d_{\log _{2}(M)}\right]=\left[b_{1}, \ldots, b_{\log _{2}(M)}\right]$ and $\mathbf{s}=162$ $\left[s_{1}, \ldots, s_{\log _{2}\left(N_{t}\right)}\right]=\left[b_{\log _{2}(M)+1}, \ldots, b_{L}\right]$. As a result, the transmit 163 bit vector can be represented as $\mathbf{b}=[\mathbf{d}, \mathbf{s}]$. Then, the first subvector 164 d is mapped to an APM symbol $s_{l}^{q}$, rather than the TA index in 165 conventional BTS-MAP. Then, the subvector $\mathbf{s}$ is transformed to a 166 new input vector by using the bit-by-bit XOR operation with jointly 167 considering the last APM-bit, which is represented as

$$
\begin{aligned}
\mathbf{s}^{\prime} & =\left[s_{1}^{\prime}, s_{2}^{\prime}, \ldots, s_{\log _{2}\left(N_{t}\right)}^{\prime}\right] \\
& =\left[d_{\log 2(M)} \oplus s_{1}, s_{1} \oplus s_{2}, \ldots, s_{\log _{2}\left(N_{t}\right)-1} \oplus s_{\log _{2}\left(N_{t}\right)}\right] \\
& =\left[b_{\log _{2}(M)} \oplus b_{\log _{2}(M)+1}, \ldots, b_{\log _{2}(L)-1} \oplus b_{\log _{2}(L)}\right]
\end{aligned}
$$

where " $\oplus$ " denotes the XOR operation. Then, the bit vector $\mathbf{s}^{\prime}$ is 169 mapped to a specific TA index of $q=\left(\sum_{k=1}^{\log _{2}\left(N_{t}\right)} 2^{\log _{2}\left(N_{t}\right)-k} s_{k}^{\prime}\right)+1,170$ which is used for transmitting the APM symbol.

The rationale of introducing the XOR operation in (2) is to increase 172 the HDS, while decreasing the HDD. To be specific, the last APM- 173 bit $b_{\log _{2}(M)}$ of $\mathbf{d}$ and the subvector $\mathbf{s}=\left[b_{\log _{2}(M)+1}, \ldots, b_{L}\right]$ form a 174 new vector $\tilde{\mathbf{s}}=\left[b_{\log _{2}(M)}, \mathbf{s}\right]$ for generating the TA-bits in (2), where 175 we have $b_{\log _{2}(M)}=0$ or $b_{\log _{2}(M)}=1$. Assuming that there are two 176 subvectors $\mathbf{s}_{a}$ and $\mathbf{s}_{b}$ and then provided that two different subvectors 177 


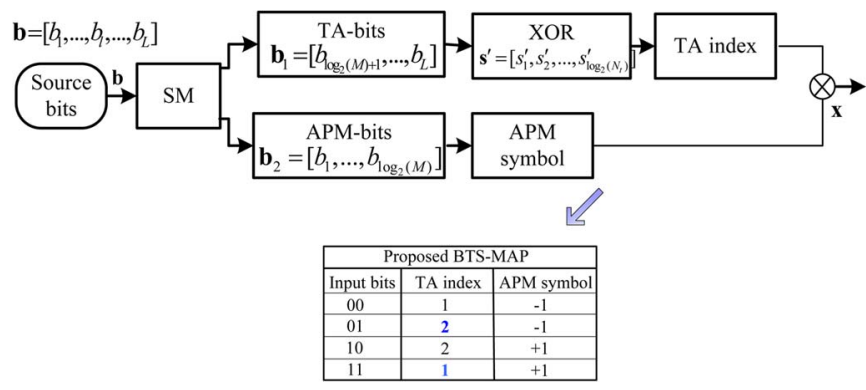

Fig. 2. Proposed BTS-MAP for SM.

$178 \tilde{\mathbf{s}}_{a}=\left[0, \mathbf{s}_{a}\right]$ and $\tilde{\mathbf{s}}_{b}=\left[1, \mathbf{s}_{b}\right]$ map to the same TA index, the HD $179 \varepsilon\left(\tilde{\mathbf{s}}_{a}, \tilde{\mathbf{s}}_{b}\right)$ is maximized to $\log _{2}\left(N_{t}\right)+1$. For example, the vectors $180 \tilde{\mathbf{s}}_{a}=[0,0,0]$ and $\tilde{\mathbf{s}}_{b}=[1,1,1]$ are mapped to the same TA index for $181 \mathrm{SM}$ using $N_{t}=4$ TAs. This result implies that the XOR operation 182 maps the subvector pair $\tilde{\mathbf{s}}_{a}$ and $\tilde{\mathbf{s}}_{b}$ having the highest HD to the same 183 TA, and hence, it is capable of increasing the HDS, while decreasing 184 the HDD.

\section{B. Example and Selection Criterion}

186 Compared with Fig. 1, we present the new BTS-MAP table in 187 Fig. 2 for the simple BPSK-modulated $(2 \times 1)$-element SM example 188 mentioned in Section II, where the HDD is reduced to 1 and the HDS 189 is increased to 2. This BTS-MAP may be more suitable for the channel 190 scenario (2) in Fig. 1, because it performs a Gray mapping when 191 considering the adjacent constellation points $-h_{1}$ and $h_{2}$ associated 192 with the MED.

193 As indicated in Section II, the design of BTS-MAP depends on the 194 distribution of MED, which can be encountered either on the same 195 TA or on different TAs. To be specific, for a given channel matrix $\mathbf{H}$, 196 the MED between the SM symbol pair $\left(\mathbf{x}_{i}, \mathbf{x}_{j}\right)$ of different TAs is 197 given by

$$
\begin{aligned}
d_{\min }^{\mathrm{Diff}}(\mathbf{H}) & =\min _{\substack{\mathbf{x}_{i}, \mathbf{x}_{j} \in \mathbb{X}, \mathbf{x}_{i} \neq \mathbf{x}_{j}, i \neq j}}\left\|\mathbf{H}\left(\mathbf{x}_{i}-\mathbf{x}_{j}\right)\right\|_{F} \\
& =\min _{s_{i}^{m}, s_{j}^{k} \in \mathbb{Q}, i \neq j}\left\|\left(\mathbf{h}_{i} s_{i}^{m}-\mathbf{h}_{j} s_{j}^{k}\right)\right\|
\end{aligned}
$$

198 where $\mathbb{X}$ is the set of all legitimate transmit symbols, $\mathbf{h}_{i}$ is the $i$ th 199 column of $\mathbf{H}$, and $s_{i}^{m}$ and $s_{j}^{k}$ represent the classic APM constellation 200 points from the set $\mathbb{Q}$. Moreover, the MED between the symbol pair of 201 the same TA is defined as

$$
d_{\min }^{\text {Same }}(\mathbf{H})=\min _{\substack{s_{i}^{m}, x_{i}^{k} \in \mathbb{Q}, s_{i}^{m} \neq s_{i}^{k}}}\left\|\mathbf{h}_{i}\left(s_{i}^{m}-s_{i}^{k}\right)\right\| .
$$

202 Based on (3) and (4), the probability of the MED $d_{\min }$ encountered 203 on different TAs is defined as $P_{\text {Diff }}=P\left(d_{\min }^{\text {Diff }}(\mathbf{H})<d_{\min }^{\text {Same }}(\mathbf{H})\right)$, 204 whereas the probability of the MED $d_{\min }$ on the same TA is defined 205 as $P_{\text {Same }}=P\left(d_{\mathrm{min}}^{\mathrm{Diff}}(\mathbf{H})>d_{\mathrm{min}}^{\mathrm{Same}}(\mathbf{H})\right)$. To minimize the probability 206 of having a bit error, the HD of the more likely adjacent constellation 207 points having the MED should be lower than that of other points lo208 cated at a higher distance than the MED. This is also the basic concept 209 of Gray mapping for a 2-D APM constellation. In the proposed BTS210 MAP, the HDD is lower than the HDS, and hence, it is more suitable 211 for the specific scenario, when the MED occurs more often in the 212 context of different TAs, which can be expressed as $P_{\text {Diff }}>P_{\text {Same }}$. In 213 other words, when we have $P_{\text {Diff }}>P_{\text {Same }}$ for an SM setup, most of 214 the error events are typically imposed by the SM symbols of different 215 TAs; hence, the minimization of the HD between these nearest points (i.e., the HDD) leads to directly minimizing the probability of bit 216 errors. Based on this observation, the BTS-MAP selection criterion 217 conceived for a specific SM scheme is formulated as follows.

Selection Criterion: If $P_{\text {Diff }}>P_{\text {Same }}$ (or $P_{\text {Diff }} \geq 1 / 2$ ) is satisfied 219 by a specific SM, then the proposed BTS-MAP is superior to the 220 conventional one in terms of reducing the BER. Otherwise, the con- 221 ventional BTS-MAP is preferred.

\section{Theoretical Analysis And Mapping Optimization}

223

Here, the probabilities of $P_{\text {Diff }}$ and $P_{\text {Same }}$ are derived, which are 224 used as an evaluation criterion for selecting a meritorious BTS-MAP 225 for a specific SM setup. As shown in [4] and [10], the phase-shift 226 keying (PSK) modulation schemes are preferred in SM; hence, PSK 227 is adopted for our theoretical analysis.

\section{A. M-PSK-Modulated $(2 \times 1)$-Element $S M$}

For the $M$-PSK-modulated $(2 \times 1)$-element SM, the associated 230 channel matrix can be expressed as $\mathbf{H}=\left[h_{1}, h_{2}\right]$, where $h_{1}$ and $h_{2}$ are 231 the fading coefficients of the first and second TAs, respectively, which 232 have zero mean and unit variance. The corresponding MED $d_{\min }^{\mathrm{Diff}}(\mathbf{H}) 233$ of (3) and the MED $d_{\mathrm{min}}^{\mathrm{Same}}(\mathbf{H})$ of (4) are given by

$$
\left\{\begin{array}{l}
d_{\min }^{\mathrm{Same}}(\mathbf{H})=\min \left\{2 \sin \left(\frac{\pi}{M}\right)\left|h_{1}\right|, 2 \sin \left(\frac{\pi}{M}\right)\left|h_{2}\right|\right\} \\
d_{\min }^{\mathrm{Diff}}(\mathbf{H})=\min \left\{\left|h_{1} e^{j \frac{2 k \pi}{M}}-h_{2}\right|, k=0, \ldots, M-1\right\} .
\end{array}\right.
$$

Now, we can derive the distribution functions of $d_{\min }^{\mathrm{Diff}}(\mathbf{H})$ and 235 $d_{\text {min }}^{\text {Same }}(\mathbf{H})$. Since the amplitudes of $h_{i}(i=1,2)$ obey the Rayleigh 236 distribution having probability density functions (PDFs) of $f_{\left|h_{i}\right|}(x)=237$ $x e^{-\left(x^{2} / 2\right)}, i=1,2$, the cumulative distribution functions (CDFs) of 238 $\eta_{i}=2 \sin (\pi / M)\left|h_{i}\right|, i=1,2$ are formulated as

$$
\begin{aligned}
F_{\eta_{i}}(x) & =\int_{0}^{\frac{x}{2 \sin \left(\frac{\pi}{M}\right)} x e^{-\frac{x^{2}}{2}} d x} \\
& =1-e^{-\frac{x^{2}}{8 \sin ^{2}\left(\frac{\pi}{M}\right)}}, \quad x \geq 0 ; i=1,2 .
\end{aligned}
$$

Based on the distribution function of $\eta_{i}$ in (6), the CDF and the 240 PDF of the random variable $d_{\mathrm{min}}^{\mathrm{Same}}(\mathbf{H})=\min \left\{\eta_{i}, i=1,2\right\}$ in (5) are 241 given by

$$
\begin{aligned}
& F_{d_{\min }^{\operatorname{Same}}(\mathbf{H})}(x)=1-e^{-\frac{x^{2}}{4 \sin ^{2}\left(\frac{\pi}{M}\right)}}, \quad x \geq 0 \\
& f_{\substack{\min \\
\text { Same }}}(\mathbf{H})=\frac{d\left[F_{d_{\min }^{\operatorname{Same}}(\mathbf{H})}(x)\right]}{d x} \\
& =\frac{x}{2 \sin ^{2}\left(\frac{\pi}{M}\right)} e^{-\frac{x^{2}}{4 \sin ^{2}\left(\frac{\pi}{M}\right)}}, \quad x \geq 0 .
\end{aligned}
$$

Let us now derive the PDF of the variable $d_{\mathrm{min}}^{\text {Diff }}(\mathbf{H})$. Since the 243 amplitudes of $\beta_{k}=\left|h_{1} e^{j(2 k \pi / M)}-h_{2}\right|, k=1, \ldots, M-1$, obey the 244 Rayleigh distribution having PDFs of $f_{\beta_{k}}(x)=(x / 2) e^{-\left(x^{2} / 4\right)}, k=245$ $1, \ldots, M-1$, the associated CDFs are 246

$$
\begin{aligned}
F_{\beta_{k}}(x) & =\int_{0}^{x} f_{\beta_{k}}(x) d x \\
& =1-e^{-\frac{x^{2}}{4}}, \quad x \geq 0 ; k=1, \ldots, M-1 .
\end{aligned}
$$


247 Based on the theory of order statistics, the CDF and the PDF of $248 d_{\min }^{\text {Diff }}(\mathbf{H})=\min \left\{\beta_{k}, k=1, \ldots, M-1\right\}$ are

$$
\begin{aligned}
F_{d_{\text {min }}^{\text {Diff }}(\mathbf{H})}(x) & =1-\left(1-\left(1-e^{-\frac{x^{2}}{4}}\right)\right)^{M} \\
& =1-e^{-\frac{M x^{2}}{4}}, \quad x \geq 0 \\
f_{d_{\min }^{\text {Diff }}(\mathbf{H})}(x) & =\frac{d\left[F_{d_{\min }^{\text {Diff }}(\mathbf{H})}(x)\right]}{d x} \\
& =\frac{M}{2} x e^{-\frac{M x^{2}}{4}}, \quad x \geq 0 .
\end{aligned}
$$

249 As illustrated in Section III, for a fixed channel matrix $250 \mathrm{H}$-provided that the proposed BTS-MAP performs better than 251 the conventional BTS-MAP-the inequality $P_{\text {Diff }}=P\left\{d_{\min }^{\mathrm{Same}}(\mathbf{H})>\right.$ $\left.252 d_{\min }^{\text {Diff }}(\mathbf{H})\right\}>1 / 2$ should be satisfied, which is equivalent to $P\{z \leq$ $2530\}>1 / 2$, where $z=d_{\min }^{\text {Diff }}(\mathbf{H})-d_{\min }^{\mathrm{Same}}(\mathbf{H})$. Based on (8) and 254 (11), the probability $P_{\text {Same }}=P(z>0)$ for the $M$-PSK-modulated $255(2 \times 1)$-element $\mathrm{SM}$ is given by

$$
\begin{aligned}
P_{\text {Same }}= & P(z>0) \\
= & \int_{0}^{\infty}\left[\int_{0}^{+\infty} f_{d_{\min }^{\text {Diff }}(\mathbf{H})}(z+y) f_{d_{\min }^{\text {Same }}(\mathbf{H})}(y) d y\right] d z \\
= & \int_{0}^{\infty} \int_{0}^{+\infty}\left(\frac{M(z+y)}{2} e^{-\frac{M(z+y)^{2}}{4}}\right) \\
& \cdot\left(\frac{y}{2 \sin ^{2}\left(\frac{\pi}{M}\right)} e^{\left.-\frac{y^{2}}{4 \sin ^{2}\left(\frac{\pi}{M}\right)}\right)}\right) d y d z \\
= & \frac{1}{\left(M \sin ^{2}\left(\frac{\pi}{M}\right)+1\right)} .
\end{aligned}
$$

256 Due to the constraint of $P_{\text {Diff }}+P_{\text {Same }}=1$, the probability $P_{\text {Diff }}=$ $257 P(z \leq 0)$ is calculated as

$$
\begin{aligned}
P_{\text {Diff }} & =P(z \leq 0)=1-P(z>0) \\
& =1-\frac{1}{M \sin ^{2}\left(\frac{\pi}{M}\right)+1}
\end{aligned}
$$

258 According to (13), the values of $P_{\text {Diff }}$ for BPSK, QPSK, 8-PSK, 259 and 16-PSK are $0.67,0.67,0.54$, and 0.39 , respectively. The result 260 in (13) indicates that $P_{\text {Diff }}>P_{\text {Same }}$ is satisfied for $M \leq 8$. In this 261 case, the proposed BTS-MAP performs better than the conventional 262 scheme.

\section{B. BPSK-Modulated $(4 \times 1)$-Element $S M$}

264 Next, consider the case of $N_{t}>2$. Here, we investigate the $(4 \times 1)$ 265 element SM using BPSK. Let us denote the channel coefficients by $266 \mathbf{H}=\left[h_{1}, h_{2}, h_{3}, h_{4}\right]$. In this system, the MEDs of (3) and (4) can be 267 represented as

$$
\begin{array}{r}
d_{\min }^{\operatorname{Same}}(\mathbf{H})=\min \left\{2\left|h_{1}\right|, 2\left|h_{2}\right|, 2\left|h_{3}\right|, 2\left|h_{4}\right|\right\} \\
d_{\min }^{\text {Diff }}(\mathbf{H})=\min \left\{\left|h_{1} \pm h_{2}\right|,\left|h_{1} \pm h_{3}\right|,\left|h_{1} \pm h_{4}\right|, \ldots\right. \\
\left.\left|h_{2} \pm h_{3}\right|,\left|h_{2} \pm h_{4}\right|,\left|h_{3} \pm h_{4}\right|\right\}
\end{array}
$$

TABLE I

METRICS OF HDS AND HDD OF THE CONVENTIONAL BTS-MAP AND THE PROPOSED BTS-MAP OF SM FOR DIFFERENT MIMO SETUPS. Moreover, the CorResponding Metrics $P_{\text {Same }}$ AND $P_{\text {Diff }}$ ARE Also Provided

\begin{tabular}{|l|c|c|l|c|c|}
\hline$N_{t} / N_{r}$ & $\begin{array}{c}\text { APM } \\
\text { scheme }\end{array}$ & $\begin{array}{c}H D S / H D D \\
(\text { Conventional) }\end{array}$ & $\begin{array}{l}H D S / H D D \\
(\text { Proposed })\end{array}$ & $\begin{array}{c}P_{\text {Same }} \\
(\%)\end{array}$ & $\begin{array}{c}P_{\text {Diff }} \\
(\%)\end{array}$ \\
\hline $2 / 1$ & BPSK & $1.00 / 1.50$ & $2.00 / 1.00$ & 28.8 & 71.2 \\
\hline $2 / 1$ & QPSK & $1.33 / 2.00$ & $2.00 / 1.50$ & 33.3 & 66.7 \\
\hline $4 / 1$ & BPSK & $1.00 / 1.83$ & $3.00 / 1.50$ & 13.4 & 86.6 \\
\hline $4 / 1$ & QPSK & $1.33 / 2.33$ & $2.67 / 2.00$ & 14.8 & 85.2 \\
\hline $2 / 2$ & BPSK & $1.00 / 1.50$ & $2.00 / 1.00$ & 16.8 & 83.2 \\
\hline $2 / 2$ & QPSK & $1.33 / 2.00$ & $2.00 / 1.50$ & 33.2 & 66.8 \\
\hline $4 / 2$ & BPSK & $1.00 / 1.83$ & $3.00 / 1.50$ & 6.6 & 93.4 \\
\hline $4 / 2$ & QPSK & $1.33 / 2.33$ & $2.67 / 2.00$ & 13.3 & 86.7 \\
\hline
\end{tabular}

Similar to Section IV-A, if the proposed BTS-MAP outperforms 268 the conventional BTS-MAP for the fading channel, the inequality 269 $P\left\{d_{\min }^{\text {Same }}(\mathbf{H})>d_{\min }^{\text {Diff }}(\mathbf{H})\right\}$ should be satisfied. To simplify the anal- 270 ysis, we shall assume that the Euclidean distances in the receive 271 constellation are statistically independent. Strictly speaking, this is 272 not true, since the constellation points created by each channel are 273 indeed interdependent through the transmit symbols. However, based 274 on regression analysis [20], we can state that the correlation between 275 $d_{\min }^{\text {Same }}(\mathbf{H})$ and $d_{\min }^{\text {Diff }}(\mathbf{H})$ is low. Moreover, we will demonstrate using 276 Monte Carlo simulations in Table I that this assumption does not 277 impose high inaccuracy.

First, for a normalized transmit constellation, the received vectors 279 $2\left|h_{i}\right|(i=1,2,3,4)$ obey the Rayleigh distribution of

\section{0}

$$
f_{2\left|h_{i}\right|}(x)=\frac{x}{4} e^{-\frac{x^{2}}{8}} \quad(i=1,2,3,4) .
$$

Based on the theory of order statistics [20] and on the four distances 281 $2\left|h_{i}\right|(i=1,2,3,4)$ in the receive SM constellation, the CDF and the 282 $\mathrm{PDF}$ of the random variable $d_{\mathrm{min}}^{\mathrm{Same}}(\mathbf{H})$ are

$$
\begin{aligned}
F_{d_{\text {min }}^{\text {Same }}}(\mathbf{H})(x) & =1-\left[1-F_{2\left|h_{i}\right|}(x)\right]^{4} \\
& =1-e^{-\frac{x^{2}}{8} \times 4}=1-e^{-\frac{x^{2}}{2}} \\
f_{d_{\min }^{\operatorname{Same}}}(\mathbf{H})(x) & =\frac{d\left[F_{d_{\min }^{\operatorname{Same}}(\mathbf{H})}(x)\right]}{d x} \\
& =x e^{-\frac{x^{2}}{2}}, \quad x>0 .
\end{aligned}
$$

Let us now derive the PDF of the MED $d_{\min }^{\text {Diff }}(\mathbf{H})$. Since $h_{1}$ and 284 $h_{2}$ are Gaussian random variables, the PDF of $\left|h_{i} \pm h_{j}\right|(i \neq j) 285$ formulated in (15) obeys the Rayleigh distribution, which can be 286 expressed as

$$
f_{\left|h_{i}-h_{j}\right|}(x)=\frac{x}{2} e^{-\frac{x^{2}}{4}}, x \geq 0 .
$$

Then, the CDF and the PDF of the random variable $d_{\mathrm{min}}^{\mathrm{Diff}}(\mathbf{H})$ are 288 given by

$$
\begin{aligned}
& F_{d_{\text {min }}^{\text {Diff }}}(\mathbf{H})(x)=1-e^{-3 x^{2}}, \quad x>0 \\
& f_{d_{\min }^{\text {Diff }}}(\mathbf{H})(x)=\frac{d\left(F_{d_{\min }^{\text {Diff }}(\mathbf{H})}(x)\right)}{d x} \\
& =6 x e^{-3 x^{2}}, \quad x>0 .
\end{aligned}
$$


290 Similar to the $M$-PSK-modulated $(2 \times 1)$-element SM, we have the 291 following probability:

$$
\begin{aligned}
P(z= & \left.d_{\min }^{\text {Diff }}(\mathbf{H})-d_{\min }^{\text {Same }}(\mathbf{H})>0\right) \\
& =\int_{0}^{\infty} \int_{0}^{\infty} f_{d_{\min }^{\text {Diff }}(\mathbf{H})}(y+z) f_{d_{\min }^{\text {Same }}(\mathbf{H})}(y) d y d z \\
& =\int_{0}^{\infty} \int_{0}^{\infty} 6(y+z) e^{-3(y+z)^{2}} \cdot y e^{-\frac{y^{2}}{2}} d y d z \\
& =\int_{0}^{\infty} y e^{-\frac{7}{2} y^{2}} d y=\frac{1}{7} .
\end{aligned}
$$

292 From (22), we have $P_{\text {Same }}=P(z>0)=1 / 7$ and $P_{\text {Diff }}=$ $293 P(z \leq 0)=1-P(z>0)=6 / 7$, which satisfies the condition $294 P_{\text {Diff }}>P_{\text {Same }}$. Hence, for the BPSK-modulated $4 \times 1$ )-element SM, 295 the proposed BTS-MAP is preferred.

\section{C. Other MIMO Setups}

297 In case of a high modulation order $M$ and a large number of TAs $298 N_{t}$, there exist too many received distances associated with different 299 values. In this case, it may be a challenge to theoretically evaluate the 300 probability $P\left\{d_{\min }^{\text {Same }}(\mathbf{H})>d_{\min }^{\text {Diff }}(\mathbf{H})\right\}$, because the exact distribution 301 of the random variable $d_{\mathrm{min}}^{\mathrm{Diff}}(\mathbf{H})$ depends on both the channel matrix 302 and on the symbol alphabet.

303 To deal with these challenging scenarios, the statistical $P_{\text {Diff }}$ and $304 P_{\text {Same }}$ results based on Monte Carlo simulations can be invoked for 305 selecting the appropriate 3-D mapping schemes. To be specific, we 306 can create a parameter lookup table for the SM schemes associated 307 with the MIMO setups considered, similar to Table II. For a specific 308 SM transmission, we assume that the relevant statistical information, 309 concerning the fading type, the MIMO antenna setup, and the PSK 310 scheme adopted, is available for the transmitter. Then, we can use 311 this information to select the appropriate BTS-MAP scheme according 312 to the lookup table designed offline. Moreover, if we consider the 313 adaptive SM schemes of [22] and [23], we can use a feedback link 314 for appropriately selecting the BTS-MAP directly by using the infor315 mation $d_{\mathrm{min}}^{\text {Diff }}(\mathbf{H})$ and $d_{\mathrm{min}}^{\mathrm{Same}}(\mathbf{H})$. If the constraint of $P_{\text {Diff }}>P_{\text {Same }}$ $316\left(d_{\min }^{\text {Diff }}(\mathbf{H})<d_{\min }^{\text {Same }}(\mathbf{H})\right.$ for adaptive $\left.\mathrm{SM}\right)$ is satisfied for a specific 317 MIMO setup, the proposed BTS-MAP is adopted. Otherwise, the 318 conventional BTS-MAP scheme is utilized.

\section{Performance Results}

\section{A. HDD and HDS Metrics for Different BTS-MAP Schemes}

321 Here, the HDs HDD and HDS of the proposed BTS-MAP and 322 of the conventional BTS-MAP are compared under different MIMO 323 setups. The simulation setup is based on 2-4 bits/symbol transmissions 324 over independent flat Rayleigh block-fading channels. Furthermore, 325 the probabilities $P_{\text {Diff }}$ and $P_{\text {Same }}$ of the occurrence of the MED $d_{\min }$ 326 are also investigated.

327 As shown in Table I, the XOR operation of (2) allows the proposed 328 BTS-MAP scheme to achieve higher HDD and lower HDS values 329 compared with those of the conventional BTS-MAP. Moreover, the 330 inequality $P_{\text {Diff }}>P_{\text {Same }}$ is satisfied in diverse MIMO setups in 331 Table I. It means that the MED $d_{\min }$ is encountered between different 332 TAs with a high probability, and hence, the proposed BTS-MAP, which 333 has a lower HDD, is preferred. For example, $P_{\text {Diff }}$ of the SM system 334 associated with $N_{t}=4, N_{r}=1$, and BPSK modulation is higher than $33586.6 \%$, whereas the HDD is reduced from 1.83 to 1.5 by using the

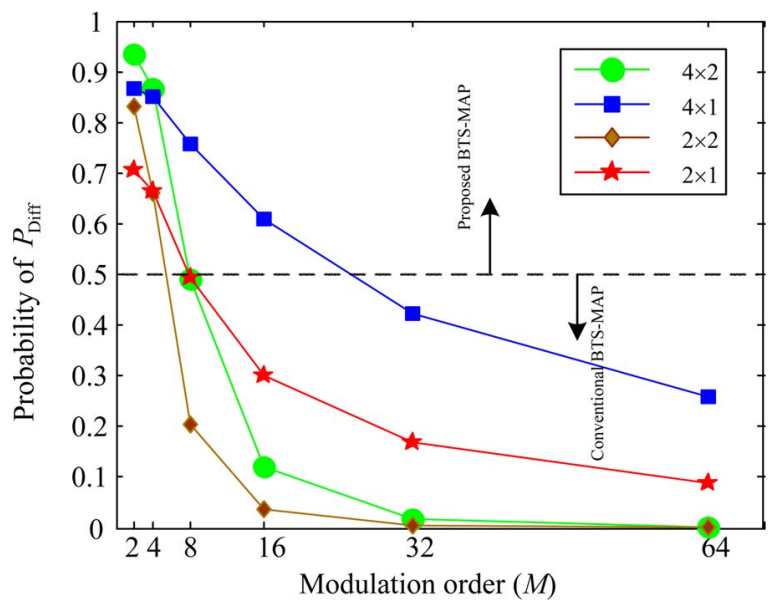

Fig. 3. Probability $P_{\text {Diff }}$ for SM under various modulation orders and different antenna configurations $N_{t} \times N_{r}$.

proposed scheme. The minimization of this HD between these nearest 336 points leads to a BER performance gain.

Moreover, Table I shows that the simulation results of $P_{\text {Diff }}$ match 338 the theoretical results for the BPSK-modulated $(2 \times 1)$ - and $(4 \times 1)-339$ element SM systems in Section IV. Note that the modest difference 340 observed between the theoretical and simulation results is due to 341 the approximation process invoked for the evaluation of $P_{\text {Diff }}$ in 342 Section IV.

Furthermore, observe in Table I that, as the modulation order 344 increases, the corresponding $P_{\text {Diff }}$ is reduced. To expound a little 345 further, we investigate the effect of the modulation order and the 346 number of TAs on the probability $P_{\text {Diff }}$ in Fig. 3. Explicitly, observe 347 in Fig. 3 that a higher modulation order may achieve a lower $P_{\text {Diff }} 348$ value for a fixed $\left(N_{t} \times N_{r}\right)$-element MIMO. This is due to the fact 349 that, if $M$ is significantly higher than $N_{t}$, the APM symbol errors 350 dominate the performance of SM. By contrast, if the number of TAs 351 $N_{t}$ is increased while maintaining a fixed value of $M$, we have an 352 increased value of $P_{\text {Diff }}$ due to the fact that the TA decision errors 353 dominate the performance of SM. Moreover, since the increase of $N_{r} 354$ can reduce both the TA and APM decision errors in SM, the specific 355 effect of this parameter depends on the particular SM setup considered. 356

As shown in Fig. 3, our BTS-MAP rule is that, if we have $P_{\text {Diff }}>357$ 0.5 , then the proposed BTS-MAP may achieve a better BER perfor- 358 mance. Otherwise, the conventional BTS-MAP can be utilized. Note 359 that, even if the statistics of $P_{\text {Diff }}$ are available for an SM-based 360 MIMO system (such as the adaptive SM of [22] and [23]), our BTS- 361 MAP selection rule still remains appropriate. Moreover, the proposed 362 scheme can be also readily extended to other types of fading channel 363 distributions, such as Rician and Nakagami fading [19].

\section{B. BER Performance}

Here, we characterize the BER performance of the proposed BTS- 366 MAP compared with the conventional BTS-MAP in MIMO Rayleigh 367 and Nakagami- $m$ fading channels. Moreover, the optimal maximum- 368 likelihood detector is adopted. Here, the notation "Pro." represents 369 the proposed BTS-MAP scheme, whereas "Con." denotes the conven- 370 tional BTS-MAP.

Fig. 4 shows the BER performance of the $(2 \times 1)$-element SM 372 systems associated with different PSK schemes. As expected, in Fig. 4, 373 the proposed BTS-MAP provides SNR gains of about $0.9 \mathrm{~dB}$ for 374 $M=2$ and $0.6 \mathrm{~dB}$ for $M=4$ at $\mathrm{BER}=10^{-2}$ over the conventional 375 BTS-MAP scheme. More important, similar to the result achieved by 376 


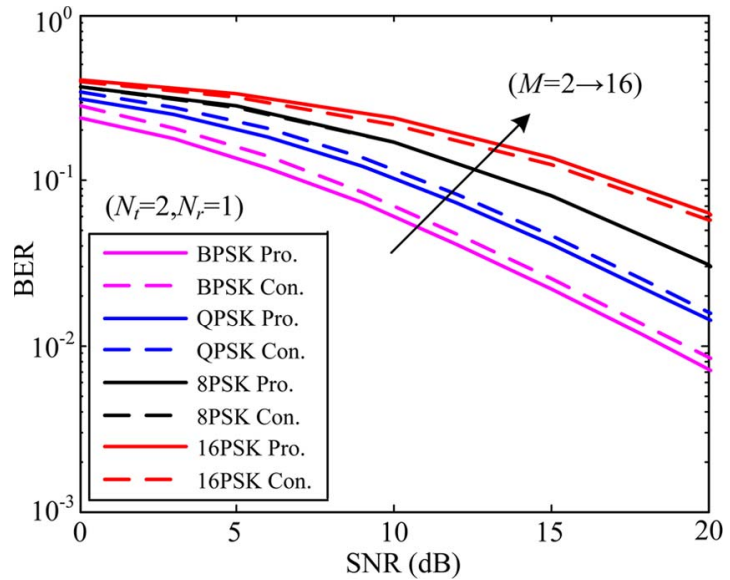

Fig. 4. BER performance of the proposed BTS-MAP and the conventional BTS-MAP schemes having $N_{t}=2, N_{r}=1$ and employing $M$-PSK signal sets.

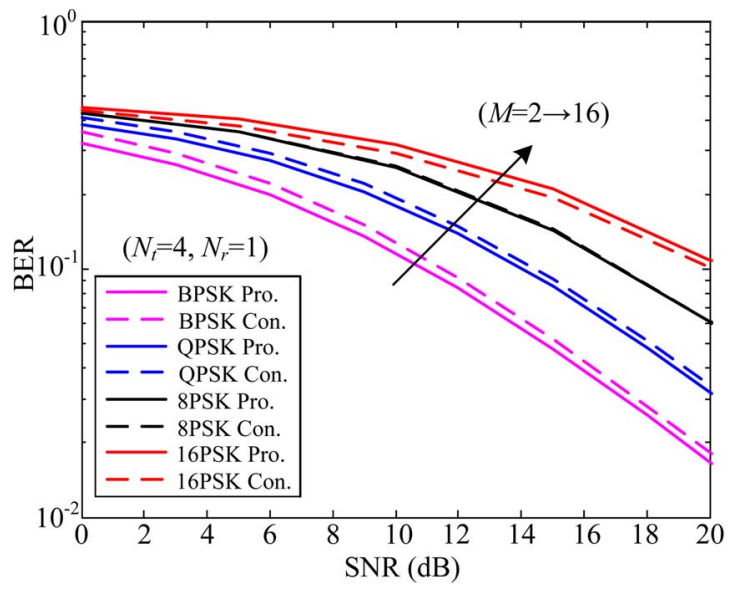

Fig. 5. BER performance of the proposed BTS-MAP and the conventional BTS-MAP schemes associated with $N_{t}=4, N_{r}=1$ and $M$-PSK schemes.

377 conventional Gray mapping for classic 2-D constellations, the specific 378 SNR value only has a modest effect on the mapping gain of the 379 proposed scheme [9]. Observe in Fig. 4 that, for the case of $M>8$, the 380 conventional BTS-MAP outperforms the proposed BTS-MAP. This 381 result is consistent with the findings in Fig. 3, where the constraint of $382 P_{\text {Diff }}>P_{\text {Same }}$ is no longer met. Additionally, for the case of $M=8$, 383 it is found that the proposed BTS-MAP and the conventional BTS384 MAP achieve almost the same BER performance. This is due to the 385 fact that, for this scheme, we have $P_{\text {Diff }} \approx 0.5$. The aforementioned 386 trends of these BTS-MAP schemes recorded for SM are also visible in 387 Fig. 5, where $(4 \times 1)$-element SM systems are considered. Moreover, 388 in Fig. 6, the performance of the proposed BTS-MAP is investigated 389 in Nakagami- $m$ fading channels. As shown in Fig. 6, the proposed 390 scheme outperforms the conventional one in $(2 \times 1)$-element MIMO 391 channels having $m=1.5$ and $m=0.8$. Since we have a higher $P_{\text {Diff }}$ 392 for the case of $m=1.5$, the corresponding BER gain is more attractive 393 than that of $m=0.8 .^{1}$

\footnotetext{
${ }^{1}$ In our simulations, the value of $P_{\text {Diff }}$ for $m=1.5$ is approximately 0.7 , whereas this value for $m=0.8$ is about 0.55 . Moreover, our proposed BTSMAP can be also directly extended to the SM in conjunction with $M$-QAM modulation. Due to space limitations, the related simulation results are not included here.
}

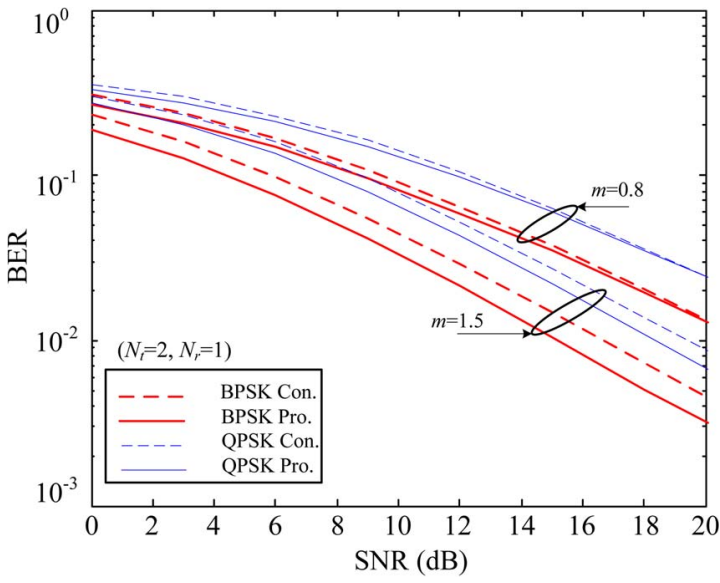

Fig. 6. BER performance of the proposed BTS-MAP and the conventional BTS-MAP schemes for $(2 \times 1)$-element Nakagami- $m$ channels.

\section{CONCLUSION}

A novel BTS-MAP scheme has been proposed for SM systems with 395 the objective of increasing the HDS and simultaneously reducing the 396 average HDD. Based on the theoretical analysis of the MED distribu- 397 tion of SM constellations, a criterion was proposed for the construction 398 of a beneficial BTS-MAP scheme for a specific MIMO setup. The 399 proposed mapping rule exhibited is attractive for employment in SM 400 systems. For achieving a further improved BER performance, our 401 further work will be focused on the integration of adaptive SM and 402 channel coding with the proposed scheme.

403

\section{REFERENCES}

[1] R. Mesleh, H. Haas, S. Sinanović, C. W. Ahn, and S. Yun, "Spatial 405 modulation," IEEE Trans. Veh. Technol., vol. 57, no. 4, pp. 2228-2241, 406 Jul. 2008.

[2] J. Jeganathan, A. Ghrayeb, L. Szczecinski, and A. Ceron, "Space shift 408 keying modulation for MIMO channels," IEEE Trans. Wireless Commun., 409 vol. 8, no. 7, pp. 3692-3703, Jul. 2009.

[3] S. Sugiura, S. Chen, and L. Hanzo, "A universal space-time architec- 411 ture for multiple-antenna aided systems," IEEE Commun. Surveys Tuts., 412 vol. 14, no. 2, pp. 401-420, 2nd Quart. 2012.

[4] M. Di Renzo, H. Haas, A. Ghrayeb, S. Sugiura, and L. Hanzo, "Spatial 414 modulation for generalized MIMO: Challenges, opportunities and imple- 415 mentation," Proc. IEEE, vol. 102, no. 1, pp. 56-103, Jan. 2014.

[5] M. Di Renzo, H. Haas, and P. M. Grant, "Spatial modulation for multiple- 417 antenna wireless systems: A survey," IEEE Commun. Mag., vol. 49, 418 no. 12, pp. 182-191, Dec. 2011.

[6] P. Yang, M. Di Renzo, Y. Xiao, S. Li, and L. Hanzo, "Design guidelines 420 for spatial modulation," IEEE Commun. Surveys Tuts., vol. 17, no. 1, 421 pp. 6-26, 1st Quart. 2015.

[7] L. Hanzo, S. X. Ng, T. Keller, and W. Webb, Quadrature Amplitude 423 Modulation: From Basics to Adaptive Trellis-Coded, Turbo-Equalised 424 and Space-Time Coded OFDM, CDMA and MC-CDMA Systems. 425 New York, NY, USA: Wiley, 2004

[8] L. Hanzo, T. H. Liew, and B. L. Yeap, Turbo Coding, Turbo Equali- 427 sation, and Space-Time Coding for Transmission over Fading Channels. 428 New York, NY, USA: Wiley-IEEE Press, 2002.

[9] E. Agreel, J. Lassing, E. G. Strom, and T. Ottosson, "Gray coding for 430 multilevel constellations in Gaussian noise," IEEE Trans. Inf. Theory, 431 vol. 53, no. 1, pp. 224-235, Jan. 2007.

[10] M. Di Renzo and H. Haas, "Bit error probability of spatial modula- 433 tion (SM-) MIMO over generalized fading channels," IEEE Trans. Veh. 434 Technol., vol. 61, no. 3, pp. 1124-1144, Mar. 2012.

[11] A. Younis, S. Sinanovic, M. Di Renzo, R. Y. Mesleh, and H. Haas, "Gen- 436 eralised sphere decoding for spatial modulation," IEEE Trans. Commun., 437 vol. 61, no. 7, pp. 2805-2815, Jul. 2013.

[12] M. Le, V. Ngo, H. Mai, X. Tran, and M. Di Renzo "Spatially modulated 439 orthogonal space-time block codes with non-vanishing determinants," 440 IEEE Trans. Commun., vol. 62, no. 1, pp. 85-98, Jan. 2014. 
442 [13] R. Y. Chang, S.-J. Lin, and W.-H. Chung, "Energy efficient transmis443 sion over space shift keying modulated MIMO channels," IEEE Trans. 444 Commun., vol. 60, no. 10, pp. 2950-2959, Oct. 2012.

445 [14] M. Di Renzo and H. Haas, "Improving the performance of space shift 446 keying (SSK) modulation via opportunistic power allocation," IEEE 447 Commun. Lett., vol. 14, no. 6, pp. 500-502, Jun. 2010.

448 [15] N. Serafimovski, M. Di Renzo, S. Sinanović, R. Y. Mesleh, and H. Haas, 449 "Fractional bit encoded spatial modulation (FBE-SM)," IEEE Commun. $450 \quad$ Lett., vol. 14, no. 5, pp. 429-431, May 2010.

451 [16] Y. Yang and S. Aïssa, "Bit-padding information guided channel hopping," 452 IEEE Commun. Lett., vol. 15, no. 2, pp. 163-165, Feb. 2011.

453 [17] R. Y. Mesleh, M. Di Renzo, H. Haas, and P. M. Grant, "Trellis coded 454 spatial modulation," IEEE Trans. Wireless Commun., vol. 9, no. 7, 455 pp. 2349-2361, Jul. 2010.

456 [18] E. Başar, Ü. Aygölü, E. Panayirci, and H. V. Poor, "New trellis code 457 design for spatial modulation," IEEE Trans. Wireless Commun., vol. 10, 458 no. 8, pp. 2670-2680, Aug. 2011.
[19] J. G. Proakis, Digital Communications, 3rd ed. Singapore: McGraw- 459 Hill, 1995.

460

[20] D. A. Freedman, Statistical Models: Theory and Practice. Cambridge, 461 U.K.: Cambridge Univ. Press, 2005.

[21] H. A. David and H. N. Nagaraja, Order Statistics, 3rd ed. New York, 463 NY, USA: Wiley, 2003.

[22] P. Yang, Y. Xiao, Y. Yi, and S. Li, "Adaptive spatial modulation for 465 wireless MIMO transmission systems," IEEE Commun. Lett., vol. 15, 466 no. 6, pp. 602-604, Jun. 2011.

467

[23] M. Maleki, H. R. Bahrami, S. Beygi, M. Kafashan, and N. H. Tran, 468 "Space modulation with CSI: Constellation design and performance eval- 469 uation," IEEE Trans. Veh. Technol., vol. 64, no. 4, pp. 1623-1634, 470 May 2013. 


\section{AUTHOR QUERIES}

\section{AUTHOR PLEASE ANSWER ALL QUERIES}

AQ1 = A citation for Table II was provided. The document, however, contains only one table. Please check. AQ2 = ML was expanded as "maximum likelihood". Please check if appropriate. Otherwise, please provide the corresponding expanded form.

AQ3 = The word "exhibit" in the sentence "The proposed mapping rule exhibits is attractive for employment in SM systems" was changed to "exhibited". Please check if appropriate. Otherwise, please make the necessary changes.

\section{END OF ALL QUERIES}




\section{Correspondence}

\section{Hybrid Bit-to-Symbol Mapping for Spatial Modulation}

2 Ping Yang, Yue Xiao, Lu Yin, Qian Tang,

3 Shaoqian Li, Senior Member, IEEE, and Lajos Hanzo, Fellow, IEEE

4 Abstract-In spatial modulation (SM), the information bit stream is di5 vided into two different sets: the transmit antenna index bits (TA-bits) and 6 the amplitude and phase modulation bits (APM-bits). However, the con7 ventional bit-to-symbol mapping (BTS-MAP) scheme maps the APM-bits 8 and the TA-bits independently. For exploiting their joint benefits, we 9 propose a new BTS-MAP rule based on the traditional 2-D Gray mapping 10 rule, which increases the Hamming distance (HD) between the symbol 11 pairs detected from the same transmit antenna (TA) and simultaneously 12 reduces the average HD between the symbol pairs gleaned from different

13 TAs. Based on the analysis of the distribution of minimum Euclidean 14 distance (MED) of SM constellations, we propose a criterion for the con15 struction of a meritorious BTS-MAP for a specific SM setup, with no need 16 for additional feedback links or extra computational complexity. Finally, 17 Monte Carlo simulations are conducted for confirming the accuracy of our 18 analysis.

19 Index Terms-Gray mapping, hamming distance (HD), multiple-input20 multiple-output (MIMO), spatial modulation (SM).

\section{INTRODUCTION}

22 Spatial modulation (SM) is a new 3-D hybrid modulation scheme 23 conceived for multiple-input-multiple-output (MIMO) transmission, 24 which exploits the indexes of the transmit antennas (TAs) as an 25 additional dimension invoked for transmitting information, apart from 26 the classic 2-D amplitude and phase modulation (APM) [1]-[5]. In $27 \mathrm{SM}$, the information bit stream is divided into two different sets: the 28 bits transmitted through the TA indexes and the APM constellations 29 [6]. For simplicity, we refer to these two sets of bits as TA-bits and 30 APM-bits.

31 In conventional 2-D APM constellations, the choice of the bit-to32 symbol mapping (BTS-MAP) rule plays an important role in determin33 ing the achievable bit error ratio (BER) performance [7]. For example, 34 an optimized BTS-MAP is capable of providing a low error floor in 35 both bit-interleaved coded modulation and in its iterative decoding and

Manuscript received July 13, 2014; revised April 23, 2015; accepted July 2, 2015. This work was supported in part by the European Research Council's Advanced Fellow Grant, by the National Science Foundation of China under Grant 61471090 , by the National High-Tech R\&D Program of China (863 Project) under Grant 2014AA01A707, and by the Open Research Fund of the National Mobile Communications Research Laboratory of Southeast University under Grant 2013D05. The review of this paper was coordinated by Dr. C. Yuen.

P. Yang, L. Yin, Q. Tang, and S. Li are with the National Key Laboratory of Science and Technology on Communications, University of Electronic Science and Technology of China, Sichuan 611731, China (e-mail: yplxw@163.com; 759562945@qq.com; qian.tang@163.com; 1sq@uestc.edu.cn).

Y. Xiao is with the National Key Laboratory of Science and Technology on Communications, University of Electronic Science and Technology of China, Sichuan 611731, China, and also with National Mobile Communications Research Laboratory, Southeast University, Nanjing 210096, China (e-mail: xiaoyue@uestc.edu.cn).

L. Hanzo is with the School of Electronics and Computer Science, University of Southampton, Southampton SO17 1BJ, U.K. (e-mail: 1h@ecs.soton.ac.uk).

Color versions of one or more of the figures in this paper are available online at http://ieeexplore.ieee.org.

Digital Object Identifier 10.1109/TVT.2015.2453216 demodulation aided counterpart (BICM-ID) [8]. It is widely known 36 that, for equally likely and statistically independent 2-D APM constel- 37 lations, Gray mapping is optimal in terms of minimizing the BER [7]. 38 In general, the optimal BTS-MAP depends on the specific geometry 39 of the signal constellation, particularly on the location of the phasors 40 separated by the minimum Euclidean distance (MED) [9]. Compared 41 with APM schemes, SM has a higher constellation dimension and a 42 different distribution of MED due to its hybrid modulation principle. 43 Hence, the classic Gray mapping proposed for 2-D constellations will 44 no longer achieve the optimal BER in fading MIMO channels.

Recently, the wide-ranging studies disseminated in [1], [4], [6], 46 and [10]-[14] have characterized some of the fundamental properties 47 of SM, such as its energy efficiency [12], [13] and the effects of 48 power imbalance [14]. In these contributions, a general framework was 49 established for the BTS-MAP rule of SM [1], where the APM-bits are 50 mapped according to the classic Gray mapping rule, whereas the TA- 51 bits are mapped to the active TA index. Due to its intuitive nature 52 and low complexity, this rule has been considered in diverse SM- 53 based systems [15]-[18]. For example, in [15] and [16], this general 54 framework was developed for an arbitrary number of TAs and hence 55 strikes a flexible tradeoff in terms of the attainable BER performance 56 and capacity. In [17] and [18], this philosophy has been extended to 57 trellis coded modulation aided SM systems for the sake of achieving 58 reliable digital transmission. However, this classic BTS-MAP scheme 59 maps the APM-bits and the TA-bits to symbols independently and 60 hence may sacrifice the classic Gray-coded benefits. Moreover, the 61 MED distribution of SM was not considered in the design process of 62 the conventional BTS-MAP scheme, which is a measure of separation 63 between two constellation points, and as a result, it has a dominant 64 influence on the BER.

Against this background, the novel contributions of this treatise are 66 as follows.

- We propose a new BTS-MAP rule based on the classic Gray- 69 coded principles for exploiting the interaction of the TA-bits 70 and the APM-bits in fading channels, which differs from the 71 conventional BTS-MAP, since it reduces the average Hamming 72 distance (HD) between the SM symbol pair of the different TAs, 73 and simultaneously, it increases the HD between the symbol pair 74 of the same TA.

- Based on the analysis of the distribution of the MED, we propose 76 a criterion for the construction of a beneficial BTS-MAP rule 77 for a specific SM-MIMO setup, which is achieved without the 78 need for an additional feedback link and with no extra compu- 79 tational complexity. Finally, performance comparisons with the 80 conventional BTS-MAP scheme of [1] are provided for different 81 constellation sizes and signal-to-noise ratios (SNRs).

Notation: $(\cdot)^{*},(\cdot)^{\mathrm{T}}$, and $(\cdot)^{\mathrm{H}}$ denote conjugate, transpose, and 83 Hermitian transpose, respectively. The probability of an event is rep- 84 resented by $P(\cdot)$. Furthermore, $\|\cdot\|$ and $|\cdot|$ denote the Euclidean 85 norm and magnitude operators, respectively; whereas $\varepsilon(\mathbf{x}, \mathbf{y})$ is the 86 HD between the binary strings $\mathbf{x}$ and $\mathbf{y} . N_{t}$ is the number of TAs, 87 and $M$ is the size of the APM constellation adopted. Let $\mathbf{b}_{i}^{m}$ be 88 the transmit bit vector mapped to the SM symbol $\mathbf{x}_{i}$ and the APM 89 symbol $s_{i}^{m}$, which corresponds to TA $i$, whereas $\mathbf{b}_{j}^{k}$ is the transmit 90 bit vector mapped to the $\mathrm{SM}$ symbol $\mathbf{x}_{j}$ related to TA $j$ and the APM 91 


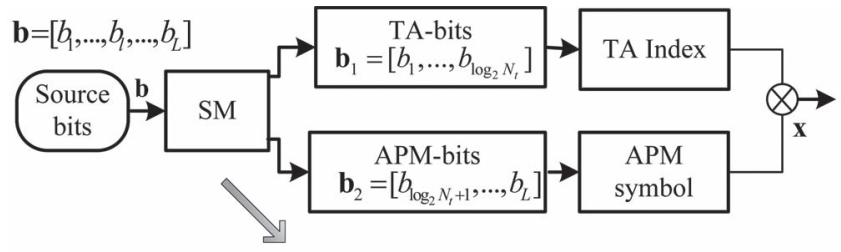

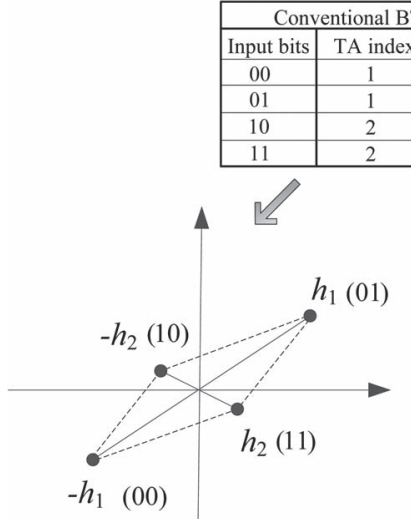

(a) $\min \left\{2\left|h_{1}\right|, 2\left|h_{2}\right|\right\}<\min \left\{\left|h_{1}-h_{2}\right|,\left|h_{1}+h_{2}\right|\right\}$
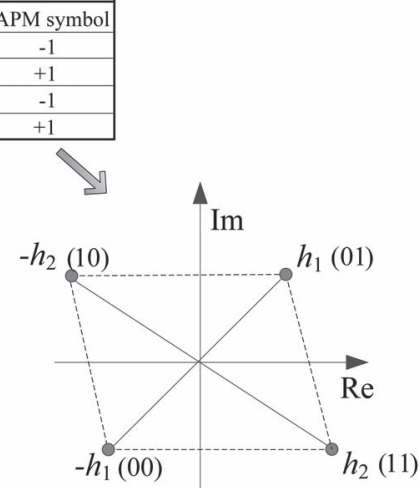

(b) $\min \left\{2\left|h_{1}\right|, 2\left|h_{2}\right|\right\} \geq \min \left\{\left|h_{1}-h_{2}\right|,\left|h_{1}+h_{2}\right|\right\}$
Fig. 1. Conventional BTS-MAP of SM: an example for the BPSK-modulated $(2 \times 1)$-element SM. (a) MED encountered on the same TA, where the conventional BTS-MAP is optimal. (b) MED encountered on different TAs, where the conventional BTS-MAP is suboptimal.

92 symbol $s_{j}^{k}$. The average HD between the symbol pair gleaned from 93 different TAs, termed as HDD, is defined as HDD $=\left(1 /\left(N_{t}\left(N_{t}-\right.\right.\right.$ 94 1) $\left.\left.M^{2}\right)\right) \sum_{i \neq j, i, j \in\left\{1, \ldots, N_{t}\right\}} \varepsilon\left(\mathbf{b}_{i}^{m}, \mathbf{b}_{j}^{k}\right)$. Moreover, the average HD 95 between the symbol pair detected from the same TA, termed as HDS, 96 is defined as HDS $=\left(1 /\left(N_{t} M(M-1)\right)\right) \sum_{i \in\left\{1, \ldots, N_{t}\right\}} \varepsilon\left(\mathbf{b}_{i}^{m}, \mathbf{b}_{i}^{k}\right)$. 97 For a fixed MIMO channel $\mathbf{H}, d_{\min }^{\mathrm{Same}}(\mathbf{H})$ is the MED between the 98 symbol pair of the same TA, $d_{\min }^{\mathrm{Diff}}(\mathbf{H})$ is the MED between the 99 symbol pair of different TAs, and the overall MED of a specific SM 100 is $d_{\min }=\min \left\{d_{\min }^{\mathrm{Same}}(\mathbf{H}), d_{\min }^{\text {Diff }}(\mathbf{H})\right\}$.

\section{II. Conventional Bit-To-Symbol MapPing Rule For 102 SPATIAL MODULATION}

103 Consider a MIMO system having $N_{t}$ transmit and $N_{r}$ receive 104 antennas. The $\left(N_{r} \times N_{t}\right)$-element channel matrix $\mathbf{H}$ is used for mod105 eling a flat-fading channel with elements having complex Gaussian 106 distributions with unit variance. We not only focus our attention on 107 the independent and identically distributed Rayleigh case but discuss 108 Nakagami- $m$ channels as well. Let $\mathbf{b}=\left[b_{1}, \ldots, b_{L}\right]$ be the transmit 109 bit vector in each time slot, which contains $L=\log _{2}\left(N_{t} M\right)$ bits. As 110 shown in Fig. 1, the input vector $\mathbf{b}$ is divided into two subvectors 111 of $\log _{2}\left(N_{t}\right)$ and $\log _{2}(M)$ bits, which are denoted by $\mathbf{b}_{1}$ and $\mathbf{b}_{2}$, 112 respectively. The bits in the subvector $\mathbf{b}_{1}$ are used for selecting a 113 unique TA index $q$ for activation, whereas the bits in the subvector $114 \mathbf{b}_{2}$ are mapped to a Gray-coded APM symbol $s_{l}^{q}$. Hence, the resultant $115 \mathrm{SM}$ symbol $\mathrm{x} \in \mathbb{C}^{N_{t} \times 1}$ is formulated as [6]

$$
\mathbf{x}=s_{l}^{q} \mathbf{e}_{q}
$$

116 where $\mathbf{e}_{q}\left(1 \leq q \leq N_{t}\right)$ is selected from the $N_{t}$-dimensional standard 117 basis vectors.

118 To expound a little further, we exemplify the binary phase-shift 119 keying (BPSK)-modulated $(2 \times 1)$-element SM in Fig. 1. As shown 120 in Fig. 1, $L=2$ input bits are divided into two single-bit streams, and 121 then, the first bit determines the activated TA (1 or 2), whereas the 122 second single bit generates the classic BPSK symbol $(+1$ or -1$)$. The aforementioned BTS-MAP method considers the APM-bits and the 123 TA-bits independently and hence facilitates a simple implementation 124 of SM.

However, this BTS-MAP method may result in a Gray-coding 126 penalty [9], which degrades the BER. Specifically, for the example 127 of BPSK-modulated $(2 \times 1)$-element SM in Fig. 1, assuming that the 128 channel matrix is $\mathbf{H}=\left[h_{1}, h_{2}\right]$, there are four received constellation 129 points denoted by $h_{1}, h_{2},-h_{1}$, and $-h_{2}$; and we investigate two 130 scenarios: 1) the MED is $d_{\min }=2\left|h_{2}\right|$; and 2) the MED is $d_{\min }=131$ $\min \left\{\left|h_{1}-h_{2}\right|,\left|h_{1}+h_{2}\right|\right\}$. Note that scenario (1) corresponds to the 132 case when the MED is encountered on the same TA (TA 2), whereas 133 scenario (2) corresponds to the case when the MED is encountered 134 on different TAs (between TAs 1 and 2). For scenario (1), the most 135 likely erroneously detected pattern is given by the nearest constellation 136 points $\left(-h_{2}, h_{2}\right)$. If the conventional BTS-MAP is adopted, these 137 points differ only in a single bit, i.e., by the difference between the 138 bits "10" and "11" in Fig. 1(a), whereas other adjacent constellation 139 points may differ in more than one bits. This mapping rule obeys 140 the concept of Gray mapping, where the probability of having a 141 bit error is minimized; hence, it has an optimal performance in this 142 specific scenario. However, for scenario (2), it is suboptimal, because 143 the nearest constellation points become $\left(-h_{1}, h_{2}\right)$, which differ in 144 two bits. This implies that more than one bit errors are associated 145 with the most likely error pattern of $\left(-h_{1}, h_{2}\right)$; hence, a performance 146 penalty will occur. This indicates that the MED distribution should be 147 considered in the design of BTS-MAP.

\section{PROPOSED BIT-TO-SYMBOL MAPPING FOR SPATIAL MODULATION}

\section{A. Principle of the Proposed BTS-MAP}

We found in Section II that the design of the BTS-MAP scheme 152 depends on the MED, which may be achieved between the symbol 153 pair gleaned from the same TA or between the symbol pair from 154 different TAs. The conventional BTS-MAP rule does not consider this 155 distribution of the MED, and hence, it becomes suboptimal for some 156 channel scenarios, as illustrated in Fig. 1(b).

For exploiting the mapping gain of SM, we propose a new 158 BTS-MAP scheme based on traditional Gray-coded modulation. 159 To be specific, similar to the conventional BTS-MAP of SM, 160 the $L$-bit input vector $\mathbf{b}=\left[b_{1}, \ldots, b_{L}\right]$ is divided into a pair 161 of subvectors $\mathbf{d}=\left[d_{1}, \ldots, d_{\log _{2}(M)}\right]=\left[b_{1}, \ldots, b_{\log _{2}(M)}\right]$ and $\mathbf{s}=162$ $\left[s_{1}, \ldots, s_{\log _{2}\left(N_{t}\right)}\right]=\left[b_{\log _{2}(M)+1}, \ldots, b_{L}\right]$. As a result, the transmit 163 bit vector can be represented as $\mathbf{b}=[\mathbf{d}, \mathbf{s}]$. Then, the first subvector 164 d is mapped to an APM symbol $s_{l}^{q}$, rather than the TA index in 165 conventional BTS-MAP. Then, the subvector $\mathbf{s}$ is transformed to a 166 new input vector by using the bit-by-bit XOR operation with jointly 167 considering the last APM-bit, which is represented as

$$
\begin{aligned}
\mathbf{s}^{\prime} & =\left[s_{1}^{\prime}, s_{2}^{\prime}, \ldots, s_{\log _{2}\left(N_{t}\right)}^{\prime}\right] \\
& =\left[d_{\log 2(M)} \oplus s_{1}, s_{1} \oplus s_{2}, \ldots, s_{\log _{2}\left(N_{t}\right)-1} \oplus s_{\log _{2}\left(N_{t}\right)}\right] \\
& =\left[b_{\log _{2}(M)} \oplus b_{\log _{2}(M)+1}, \ldots, b_{\log _{2}(L)-1} \oplus b_{\log _{2}(L)}\right]
\end{aligned}
$$

where " $\oplus$ " denotes the XOR operation. Then, the bit vector $\mathbf{s}^{\prime}$ is 169 mapped to a specific TA index of $q=\left(\sum_{k=1}^{\log _{2}\left(N_{t}\right)} 2^{\log _{2}\left(N_{t}\right)-k} s_{k}^{\prime}\right)+1,170$ which is used for transmitting the APM symbol.

The rationale of introducing the XOR operation in (2) is to increase 172 the HDS, while decreasing the HDD. To be specific, the last APM- 173 bit $b_{\log _{2}(M)}$ of $\mathbf{d}$ and the subvector $\mathbf{s}=\left[b_{\log _{2}(M)+1}, \ldots, b_{L}\right]$ form a 174 new vector $\tilde{\mathbf{s}}=\left[b_{\log _{2}(M)}, \mathbf{s}\right]$ for generating the TA-bits in (2), where 175 we have $b_{\log _{2}(M)}=0$ or $b_{\log _{2}(M)}=1$. Assuming that there are two 176 subvectors $\mathbf{s}_{a}$ and $\mathbf{s}_{b}$ and then provided that two different subvectors 177 


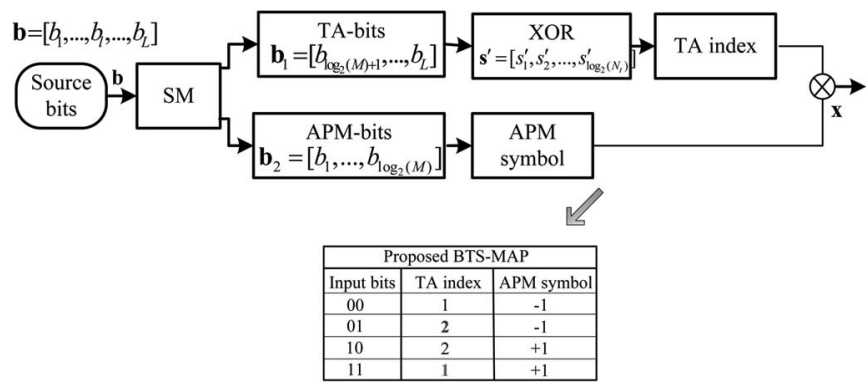

Fig. 2. Proposed BTS-MAP for SM.

$178 \tilde{\mathbf{s}}_{a}=\left[0, \mathbf{s}_{a}\right]$ and $\tilde{\mathbf{s}}_{b}=\left[1, \mathbf{s}_{b}\right]$ map to the same TA index, the HD $179 \varepsilon\left(\tilde{\mathbf{s}}_{a}, \tilde{\mathbf{s}}_{b}\right)$ is maximized to $\log _{2}\left(N_{t}\right)+1$. For example, the vectors $180 \tilde{\mathbf{s}}_{a}=[0,0,0]$ and $\tilde{\mathbf{s}}_{b}=[1,1,1]$ are mapped to the same TA index for $181 \mathrm{SM}$ using $N_{t}=4$ TAs. This result implies that the XOR operation 182 maps the subvector pair $\tilde{\mathbf{s}}_{a}$ and $\tilde{\mathbf{s}}_{b}$ having the highest HD to the same 183 TA, and hence, it is capable of increasing the HDS, while decreasing 184 the HDD.

\section{B. Example and Selection Criterion}

186 Compared with Fig. 1, we present the new BTS-MAP table in 187 Fig. 2 for the simple BPSK-modulated $(2 \times 1)$-element SM example 188 mentioned in Section II, where the HDD is reduced to 1 and the HDS 189 is increased to 2. This BTS-MAP may be more suitable for the channel 190 scenario (2) in Fig. 1, because it performs a Gray mapping when 191 considering the adjacent constellation points $-h_{1}$ and $h_{2}$ associated 192 with the MED.

193 As indicated in Section II, the design of BTS-MAP depends on the 194 distribution of MED, which can be encountered either on the same 195 TA or on different TAs. To be specific, for a given channel matrix $\mathbf{H}$, 196 the MED between the SM symbol pair $\left(\mathbf{x}_{i}, \mathbf{x}_{j}\right)$ of different TAs is 197 given by

$$
\begin{aligned}
d_{\min }^{\mathrm{Diff}}(\mathbf{H}) & =\min _{\substack{\mathbf{x}_{i}, \mathbf{x}_{j} \in \mathbb{X}, \mathbf{x}_{i} \neq \mathbf{x}_{j}, i \neq j}}\left\|\mathbf{H}\left(\mathbf{x}_{i}-\mathbf{x}_{j}\right)\right\|_{F} \\
& =\min _{s_{i}^{m}, s_{j}^{k} \in \mathbb{Q}, i \neq j}\left\|\left(\mathbf{h}_{i} s_{i}^{m}-\mathbf{h}_{j} s_{j}^{k}\right)\right\|
\end{aligned}
$$

198 where $\mathbb{X}$ is the set of all legitimate transmit symbols, $\mathbf{h}_{i}$ is the $i$ th 199 column of $\mathbf{H}$, and $s_{i}^{m}$ and $s_{j}^{k}$ represent the classic APM constellation 200 points from the set $\mathbb{Q}$. Moreover, the MED between the symbol pair of 201 the same TA is defined as

$$
d_{\min }^{\text {Same }}(\mathbf{H})=\min _{\substack{s_{i}^{m}, x_{i}^{k} \in \mathbb{Q}, s_{i}^{m} \neq s_{i}^{k}}}\left\|\mathbf{h}_{i}\left(s_{i}^{m}-s_{i}^{k}\right)\right\| .
$$

202 Based on (3) and (4), the probability of the MED $d_{\min }$ encountered 203 on different TAs is defined as $P_{\text {Diff }}=P\left(d_{\min }^{\text {Diff }}(\mathbf{H})<d_{\min }^{\text {Same }}(\mathbf{H})\right)$, 204 whereas the probability of the MED $d_{\min }$ on the same TA is defined 205 as $P_{\text {Same }}=P\left(d_{\mathrm{min}}^{\mathrm{Diff}}(\mathbf{H})>d_{\mathrm{min}}^{\mathrm{Same}}(\mathbf{H})\right)$. To minimize the probability 206 of having a bit error, the HD of the more likely adjacent constellation 207 points having the MED should be lower than that of other points lo208 cated at a higher distance than the MED. This is also the basic concept 209 of Gray mapping for a 2-D APM constellation. In the proposed BTS210 MAP, the HDD is lower than the HDS, and hence, it is more suitable 211 for the specific scenario, when the MED occurs more often in the 212 context of different TAs, which can be expressed as $P_{\text {Diff }}>P_{\text {Same }}$. In 213 other words, when we have $P_{\text {Diff }}>P_{\text {Same }}$ for an SM setup, most of 214 the error events are typically imposed by the SM symbols of different 215 TAs; hence, the minimization of the HD between these nearest points (i.e., the HDD) leads to directly minimizing the probability of bit 216 errors. Based on this observation, the BTS-MAP selection criterion 217 conceived for a specific SM scheme is formulated as follows.

Selection Criterion: If $P_{\text {Diff }}>P_{\text {Same }}$ (or $P_{\text {Diff }} \geq 1 / 2$ ) is satisfied 219 by a specific SM, then the proposed BTS-MAP is superior to the 220 conventional one in terms of reducing the BER. Otherwise, the con- 221 ventional BTS-MAP is preferred.

\section{Theoretical Analysis and Mapping Optimization}

223

Here, the probabilities of $P_{\text {Diff }}$ and $P_{\text {Same }}$ are derived, which are 224 used as an evaluation criterion for selecting a meritorious BTS-MAP 225 for a specific SM setup. As shown in [4] and [10], the phase-shift 226 keying (PSK) modulation schemes are preferred in SM; hence, PSK 227 is adopted for our theoretical analysis.

\section{A. M-PSK-Modulated $(2 \times 1)$-Element $S M$}

For the $M$-PSK-modulated $(2 \times 1)$-element $\mathrm{SM}$, the associated 230 channel matrix can be expressed as $\mathbf{H}=\left[h_{1}, h_{2}\right]$, where $h_{1}$ and $h_{2}$ are 231 the fading coefficients of the first and second TAs, respectively, which 232 have zero mean and unit variance. The corresponding MED $d_{\min }^{\mathrm{Diff}}(\mathbf{H}) 233$ of (3) and the MED $d_{\mathrm{min}}^{\mathrm{Same}}(\mathbf{H})$ of (4) are given by

$$
\left\{\begin{array}{l}
d_{\min }^{\mathrm{Same}}(\mathbf{H})=\min \left\{2 \sin \left(\frac{\pi}{M}\right)\left|h_{1}\right|, 2 \sin \left(\frac{\pi}{M}\right)\left|h_{2}\right|\right\} \\
d_{\min }^{\mathrm{Diff}}(\mathbf{H})=\min \left\{\left|h_{1} e^{j \frac{2 k \pi}{M}}-h_{2}\right|, k=0, \ldots, M-1\right\} .
\end{array}\right.
$$

Now, we can derive the distribution functions of $d_{\min }^{\mathrm{Diff}}(\mathbf{H})$ and 235 $d_{\text {min }}^{\text {Same }}(\mathbf{H})$. Since the amplitudes of $h_{i}(i=1,2)$ obey the Rayleigh 236 distribution having probability density functions (PDFs) of $f_{\left|h_{i}\right|}(x)=237$ $x e^{-\left(x^{2} / 2\right)}, i=1,2$, the cumulative distribution functions (CDFs) of 238 $\eta_{i}=2 \sin (\pi / M)\left|h_{i}\right|, i=1,2$ are formulated as

$$
\begin{aligned}
F_{\eta_{i}}(x) & =\int_{0}^{\frac{x}{2 \sin \left(\frac{\pi}{M}\right)}} x e^{-\frac{x^{2}}{2}} d x \\
& =1-e^{-\frac{x^{2}}{8 \sin ^{2}\left(\frac{\pi}{M}\right)}}, \quad x \geq 0 ; i=1,2 .
\end{aligned}
$$

Based on the distribution function of $\eta_{i}$ in (6), the CDF and the 240 PDF of the random variable $d_{\mathrm{min}}^{\mathrm{Same}}(\mathbf{H})=\min \left\{\eta_{i}, i=1,2\right\}$ in (5) are 241 given by

$$
\begin{aligned}
& F_{d_{\min }^{\operatorname{Same}}(\mathbf{H})}(x)=1-e^{-\frac{x^{2}}{4 \sin ^{2}\left(\frac{\pi}{M}\right)}}, \quad x \geq 0 \\
& f_{d_{\mathrm{min}}^{\mathrm{Same}}(\mathbf{H})}(x)=\frac{d\left[F_{d_{\mathrm{min}}^{\mathrm{Same}}(\mathbf{H})}(x)\right]}{d x} \\
& =\frac{x}{2 \sin ^{2}\left(\frac{\pi}{M}\right)} e^{-\frac{x^{2}}{4 \sin ^{2}\left(\frac{\pi}{M}\right)}}, \quad x \geq 0 .
\end{aligned}
$$

Let us now derive the PDF of the variable $d_{\mathrm{min}}^{\text {Diff }}(\mathbf{H})$. Since the 243 amplitudes of $\beta_{k}=\left|h_{1} e^{j(2 k \pi / M)}-h_{2}\right|, k=1, \ldots, M-1$, obey the 244 Rayleigh distribution having PDFs of $f_{\beta_{k}}(x)=(x / 2) e^{-\left(x^{2} / 4\right)}, k=245$ $1, \ldots, M-1$, the associated CDFs are 246

$$
\begin{aligned}
F_{\beta_{k}}(x) & =\int_{0}^{x} f_{\beta_{k}}(x) d x \\
& =1-e^{-\frac{x^{2}}{4}}, \quad x \geq 0 ; k=1, \ldots, M-1 .
\end{aligned}
$$


247 Based on the theory of order statistics, the CDF and the PDF of $248 d_{\min }^{\text {Diff }}(\mathbf{H})=\min \left\{\beta_{k}, k=1, \ldots, M-1\right\}$ are

$$
\begin{aligned}
F_{d_{\text {min }}^{\text {Diff }}(\mathbf{H})}(x) & =1-\left(1-\left(1-e^{-\frac{x^{2}}{4}}\right)\right)^{M} \\
& =1-e^{-\frac{M x^{2}}{4}}, \quad x \geq 0 \\
f_{d_{\min }^{\text {Diff }}(\mathbf{H})}(x) & =\frac{d\left[F_{d_{\min }^{\text {Diff }}(\mathbf{H})}(x)\right]}{d x} \\
& =\frac{M}{2} x e^{-\frac{M x^{2}}{4}}, \quad x \geq 0 .
\end{aligned}
$$

249 As illustrated in Section III, for a fixed channel matrix $250 \mathrm{H}$-provided that the proposed BTS-MAP performs better than 251 the conventional BTS-MAP - the inequality $P_{\text {Diff }}=P\left\{d_{\min }^{\mathrm{Same}}(\mathbf{H})>\right.$ $\left.252 d_{\min }^{\text {Diff }}(\mathbf{H})\right\}>1 / 2$ should be satisfied, which is equivalent to $P\{z \leq$ $2530\}>1 / 2$, where $z=d_{\mathrm{min}}^{\mathrm{Diff}}(\mathbf{H})-d_{\mathrm{min}}^{\mathrm{Same}}(\mathbf{H})$. Based on (8) and 254 (11), the probability $P_{\text {Same }}=P(z>0)$ for the $M$-PSK-modulated $255(2 \times 1)$-element $\mathrm{SM}$ is given by

$$
\begin{aligned}
P_{\text {Same }}= & P(z>0) \\
= & \int_{0}^{\infty}\left[\int_{0}^{+\infty} f_{d_{\min }^{\text {Diff }}(\mathbf{H})}(z+y) f_{d_{\min }^{\text {Same }}(\mathbf{H})}(y) d y\right] d z \\
= & \int_{0}^{\infty} \int_{0}^{+\infty}\left(\frac{M(z+y)}{2} e^{-\frac{M(z+y)^{2}}{4}}\right) \\
& \cdot\left(\frac{y}{2 \sin ^{2}\left(\frac{\pi}{M}\right)} e^{-\frac{y^{2}}{4 \sin ^{2}\left(\frac{\pi}{M}\right)}}\right) d y d z \\
= & \frac{1}{\left(M \sin ^{2}\left(\frac{\pi}{M}\right)+1\right)} .
\end{aligned}
$$

256 Due to the constraint of $P_{\text {Diff }}+P_{\text {Same }}=1$, the probability $P_{\text {Diff }}=$ $257 P(z \leq 0)$ is calculated as

$$
\begin{aligned}
P_{\text {Diff }} & =P(z \leq 0)=1-P(z>0) \\
& =1-\frac{1}{M \sin ^{2}\left(\frac{\pi}{M}\right)+1}
\end{aligned}
$$

258 According to (13), the values of $P_{\text {Diff }}$ for BPSK, QPSK, 8-PSK, 259 and 16-PSK are $0.67,0.67,0.54$, and 0.39 , respectively. The result 260 in (13) indicates that $P_{\text {Diff }}>P_{\text {Same }}$ is satisfied for $M \leq 8$. In this 261 case, the proposed BTS-MAP performs better than the conventional 262 scheme.

\section{B. BPSK-Modulated $(4 \times 1)$-Element $S M$}

264 Next, consider the case of $N_{t}>2$. Here, we investigate the $(4 \times 1)$ 265 element SM using BPSK. Let us denote the channel coefficients by $266 \mathbf{H}=\left[h_{1}, h_{2}, h_{3}, h_{4}\right]$. In this system, the MEDs of (3) and (4) can be 267 represented as

$$
\begin{array}{r}
d_{\min }^{\text {Same }}(\mathbf{H})=\min \left\{2\left|h_{1}\right|, 2\left|h_{2}\right|, 2\left|h_{3}\right|, 2\left|h_{4}\right|\right\} \\
d_{\min }^{\text {Diff }}(\mathbf{H})=\min \left\{\left|h_{1} \pm h_{2}\right|,\left|h_{1} \pm h_{3}\right|,\left|h_{1} \pm h_{4}\right|, \ldots\right. \\
\left.\left|h_{2} \pm h_{3}\right|,\left|h_{2} \pm h_{4}\right|,\left|h_{3} \pm h_{4}\right|\right\}
\end{array}
$$

TABLE I

METRICS OF HDS AND HDD OF THE CONVENTIONAL BTS-MAP AND THE PROPOSED BTS-MAP OF SM FOR DIFFERENT MIMO SETUPS. Moreover, the CORRESPONDING METRICS $P_{\text {Same }}$ AND $P_{\text {Diff }}$ ARE Also Provided

\begin{tabular}{|l|c|c|l|c|c|}
\hline$N_{t} / N_{r}$ & $\begin{array}{c}\text { APM } \\
\text { scheme }\end{array}$ & $\begin{array}{c}H D S / H D D \\
\text { (Conventional) }\end{array}$ & $\begin{array}{l}H D S / H D D \\
\text { (Proposed) }\end{array}$ & $\begin{array}{c}P_{\text {Same }} \\
(\%)\end{array}$ & $\begin{array}{c}P_{\text {Diff }} \\
(\%)\end{array}$ \\
\hline $2 / 1$ & BPSK & $1.00 / 1.50$ & $2.00 / 1.00$ & 28.8 & 71.2 \\
\hline $2 / 1$ & QPSK & $1.33 / 2.00$ & $2.00 / 1.50$ & 33.3 & 66.7 \\
\hline $4 / 1$ & BPSK & $1.00 / 1.83$ & $3.00 / 1.50$ & 13.4 & 86.6 \\
\hline $4 / 1$ & QPSK & $1.33 / 2.33$ & $2.67 / 2.00$ & 14.8 & 85.2 \\
\hline $2 / 2$ & BPSK & $1.00 / 1.50$ & $2.00 / 1.00$ & 16.8 & 83.2 \\
\hline $2 / 2$ & QPSK & $1.33 / 2.00$ & $2.00 / 1.50$ & 33.2 & 66.8 \\
\hline $4 / 2$ & BPSK & $1.00 / 1.83$ & $3.00 / 1.50$ & 6.6 & 93.4 \\
\hline $4 / 2$ & QPSK & $1.33 / 2.33$ & $2.67 / 2.00$ & 13.3 & 86.7 \\
\hline
\end{tabular}

Similar to Section IV-A, if the proposed BTS-MAP outperforms 268 the conventional BTS-MAP for the fading channel, the inequality 269 $P\left\{d_{\min }^{\text {Same }}(\mathbf{H})>d_{\min }^{\text {Diff }}(\mathbf{H})\right\}$ should be satisfied. To simplify the anal- 270 ysis, we shall assume that the Euclidean distances in the receive 271 constellation are statistically independent. Strictly speaking, this is 272 not true, since the constellation points created by each channel are 273 indeed interdependent through the transmit symbols. However, based 274 on regression analysis [20], we can state that the correlation between 275 $d_{\min }^{\text {Same }}(\mathbf{H})$ and $d_{\min }^{\text {Diff }}(\mathbf{H})$ is low. Moreover, we will demonstrate using 276 Monte Carlo simulations in Table I that this assumption does not 277 impose high inaccuracy.

First, for a normalized transmit constellation, the received vectors 279 $2\left|h_{i}\right|(i=1,2,3,4)$ obey the Rayleigh distribution of

280

$$
f_{2\left|h_{i}\right|}(x)=\frac{x}{4} e^{-\frac{x^{2}}{8}} \quad(i=1,2,3,4) .
$$

Based on the theory of order statistics [20] and on the four distances 281 $2\left|h_{i}\right|(i=1,2,3,4)$ in the receive SM constellation, the CDF and the 282 $\mathrm{PDF}$ of the random variable $d_{\mathrm{min}}^{\mathrm{Same}}(\mathbf{H})$ are

$$
\begin{aligned}
F_{d_{\text {min }}^{\text {Same }}}(\mathbf{H})(x) & =1-\left[1-F_{2\left|h_{i}\right|}(x)\right]^{4} \\
& =1-e^{-\frac{x^{2}}{8} \times 4}=1-e^{-\frac{x^{2}}{2}} \\
f_{d_{\text {min }}^{\text {Same }}}(\mathbf{H})(x) & =\frac{d\left[F_{d_{\min }^{\operatorname{Same}}(\mathbf{H})}(x)\right]}{d x} \\
& =x e^{-\frac{x^{2}}{2}}, \quad x>0 .
\end{aligned}
$$

Let us now derive the PDF of the $\operatorname{MED} d_{\min }^{\text {Diff }}(\mathbf{H})$. Since $h_{1}$ and 284 $h_{2}$ are Gaussian random variables, the $\mathrm{PDF}$ of $\left|h_{i} \pm h_{j}\right|(i \neq j) 285$ formulated in (15) obeys the Rayleigh distribution, which can be 286 expressed as

$$
f_{\left|h_{i}-h_{j}\right|}(x)=\frac{x}{2} e^{-\frac{x^{2}}{4}}, x \geq 0 .
$$

Then, the CDF and the PDF of the random variable $d_{\min }^{\text {Diff }}(\mathbf{H})$ are 288 given by

$$
\begin{aligned}
& F_{d_{\min }^{\text {Diff }}}(\mathbf{H})(x)=1-e^{-3 x^{2}}, \quad x>0 \\
& f_{d \underset{\text { min }}{\text { Diff }}}(\mathbf{H})(x)=\frac{d\left(F_{d_{\min }^{\text {Diff }}(\mathbf{H})}(x)\right)}{d x} \\
& =6 x e^{-3 x^{2}}, \quad x>0 .
\end{aligned}
$$


290 Similar to the $M$-PSK-modulated $(2 \times 1)$-element SM, we have the 291 following probability:

$$
\begin{aligned}
P(z= & \left.d_{\min }^{\text {Diff }}(\mathbf{H})-d_{\min }^{\text {Same }}(\mathbf{H})>0\right) \\
& =\int_{0}^{\infty} \int_{0}^{\infty} f_{d_{\min }^{\text {Diff }}(\mathbf{H})}(y+z) f_{d_{\min }^{\text {Same }}(\mathbf{H})}(y) d y d z \\
& =\int_{0}^{\infty} \int_{0}^{\infty} 6(y+z) e^{-3(y+z)^{2}} \cdot y e^{-\frac{y^{2}}{2}} d y d z \\
& =\int_{0}^{\infty} y e^{-\frac{7}{2} y^{2}} d y=\frac{1}{7} .
\end{aligned}
$$

292 From (22), we have $P_{\text {Same }}=P(z>0)=1 / 7$ and $P_{\text {Diff }}=$ $293 P(z \leq 0)=1-P(z>0)=6 / 7$, which satisfies the condition $294 P_{\text {Diff }}>P_{\text {Same }}$. Hence, for the BPSK-modulated $4 \times 1$ )-element SM, 295 the proposed BTS-MAP is preferred.

\section{C. Other MIMO Setups}

297 In case of a high modulation order $M$ and a large number of TAs $298 N_{t}$, there exist too many received distances associated with different 299 values. In this case, it may be a challenge to theoretically evaluate the 300 probability $P\left\{d_{\min }^{\text {Same }}(\mathbf{H})>d_{\min }^{\text {Diff }}(\mathbf{H})\right\}$, because the exact distribution 301 of the random variable $d_{\mathrm{min}}^{\mathrm{Diff}}(\mathbf{H})$ depends on both the channel matrix 302 and on the symbol alphabet.

303 To deal with these challenging scenarios, the statistical $P_{\text {Diff }}$ and $304 P_{\text {Same }}$ results based on Monte Carlo simulations can be invoked for 305 selecting the appropriate 3-D mapping schemes. To be specific, we 306 can create a parameter lookup table for the SM schemes associated 307 with the MIMO setups considered, similar to Table II. For a specific 308 SM transmission, we assume that the relevant statistical information, 309 concerning the fading type, the MIMO antenna setup, and the PSK 310 scheme adopted, is available for the transmitter. Then, we can use 311 this information to select the appropriate BTS-MAP scheme according 312 to the lookup table designed offline. Moreover, if we consider the 313 adaptive SM schemes of [22] and [23], we can use a feedback link 314 for appropriately selecting the BTS-MAP directly by using the infor315 mation $d_{\mathrm{min}}^{\text {Diff }}(\mathbf{H})$ and $d_{\mathrm{min}}^{\mathrm{Same}}(\mathbf{H})$. If the constraint of $P_{\text {Diff }}>P_{\text {Same }}$ $316\left(d_{\min }^{\text {Diff }}(\mathbf{H})<d_{\min }^{\text {Same }}(\mathbf{H})\right.$ for adaptive $\left.\mathrm{SM}\right)$ is satisfied for a specific 317 MIMO setup, the proposed BTS-MAP is adopted. Otherwise, the 318 conventional BTS-MAP scheme is utilized.

\section{Performance Results}

\section{A. HDD and HDS Metrics for Different BTS-MAP Schemes}

321 Here, the HDs HDD and HDS of the proposed BTS-MAP and 322 of the conventional BTS-MAP are compared under different MIMO 323 setups. The simulation setup is based on 2-4 bits/symbol transmissions 324 over independent flat Rayleigh block-fading channels. Furthermore, 325 the probabilities $P_{\text {Diff }}$ and $P_{\text {Same }}$ of the occurrence of the MED $d_{\min }$ 326 are also investigated.

327 As shown in Table I, the XOR operation of (2) allows the proposed 328 BTS-MAP scheme to achieve higher HDD and lower HDS values 329 compared with those of the conventional BTS-MAP. Moreover, the 330 inequality $P_{\text {Diff }}>P_{\text {Same }}$ is satisfied in diverse MIMO setups in 331 Table I. It means that the MED $d_{\min }$ is encountered between different 332 TAs with a high probability, and hence, the proposed BTS-MAP, which 333 has a lower HDD, is preferred. For example, $P_{\text {Diff }}$ of the SM system 334 associated with $N_{t}=4, N_{r}=1$, and BPSK modulation is higher than $33586.6 \%$, whereas the HDD is reduced from 1.83 to 1.5 by using the

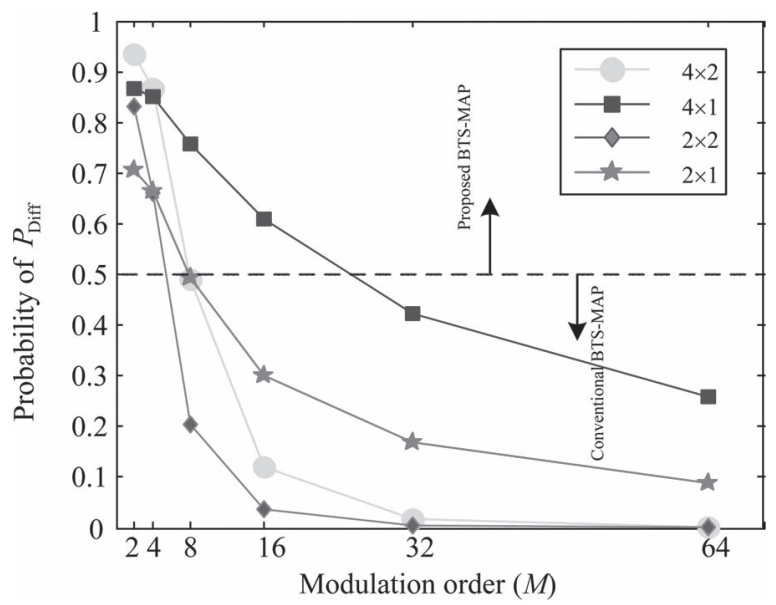

Fig. 3. Probability $P_{\text {Diff }}$ for SM under various modulation orders and different antenna configurations $N_{t} \times N_{r}$.

proposed scheme. The minimization of this HD between these nearest 336 points leads to a BER performance gain.

Moreover, Table I shows that the simulation results of $P_{\text {Diff }}$ match 338 the theoretical results for the BPSK-modulated $(2 \times 1)$ - and $(4 \times 1)-339$ element SM systems in Section IV. Note that the modest difference 340 observed between the theoretical and simulation results is due to 341 the approximation process invoked for the evaluation of $P_{\text {Diff }}$ in 342 Section IV.

Furthermore, observe in Table I that, as the modulation order 344 increases, the corresponding $P_{\text {Diff }}$ is reduced. To expound a little 345 further, we investigate the effect of the modulation order and the 346 number of TAs on the probability $P_{\text {Diff }}$ in Fig. 3. Explicitly, observe 347 in Fig. 3 that a higher modulation order may achieve a lower $P_{\text {Diff }} 348$ value for a fixed $\left(N_{t} \times N_{r}\right)$-element MIMO. This is due to the fact 349 that, if $M$ is significantly higher than $N_{t}$, the APM symbol errors 350 dominate the performance of SM. By contrast, if the number of TAs 351 $N_{t}$ is increased while maintaining a fixed value of $M$, we have an 352 increased value of $P_{\text {Diff }}$ due to the fact that the TA decision errors 353 dominate the performance of SM. Moreover, since the increase of $N_{r} 354$ can reduce both the TA and APM decision errors in SM, the specific 355 effect of this parameter depends on the particular SM setup considered. 356

As shown in Fig. 3, our BTS-MAP rule is that, if we have $P_{\text {Diff }}>357$ 0.5 , then the proposed BTS-MAP may achieve a better BER perfor- 358 mance. Otherwise, the conventional BTS-MAP can be utilized. Note 359 that, even if the statistics of $P_{\text {Diff }}$ are available for an SM-based 360 MIMO system (such as the adaptive SM of [22] and [23]), our BTS- 361 MAP selection rule still remains appropriate. Moreover, the proposed 362 scheme can be also readily extended to other types of fading channel 363 distributions, such as Rician and Nakagami fading [19].

\section{B. BER Performance}

Here, we characterize the BER performance of the proposed BTS- 366 MAP compared with the conventional BTS-MAP in MIMO Rayleigh 367 and Nakagami- $m$ fading channels. Moreover, the optimal maximum- 368 likelihood detector is adopted. Here, the notation "Pro." represents 369 the proposed BTS-MAP scheme, whereas "Con." denotes the conven- 370 tional BTS-MAP.

Fig. 4 shows the BER performance of the $(2 \times 1)$-element SM 372 systems associated with different PSK schemes. As expected, in Fig. 4, 373 the proposed BTS-MAP provides SNR gains of about $0.9 \mathrm{~dB}$ for 374 $M=2$ and $0.6 \mathrm{~dB}$ for $M=4$ at $\mathrm{BER}=10^{-2}$ over the conventional 375 BTS-MAP scheme. More important, similar to the result achieved by 376 


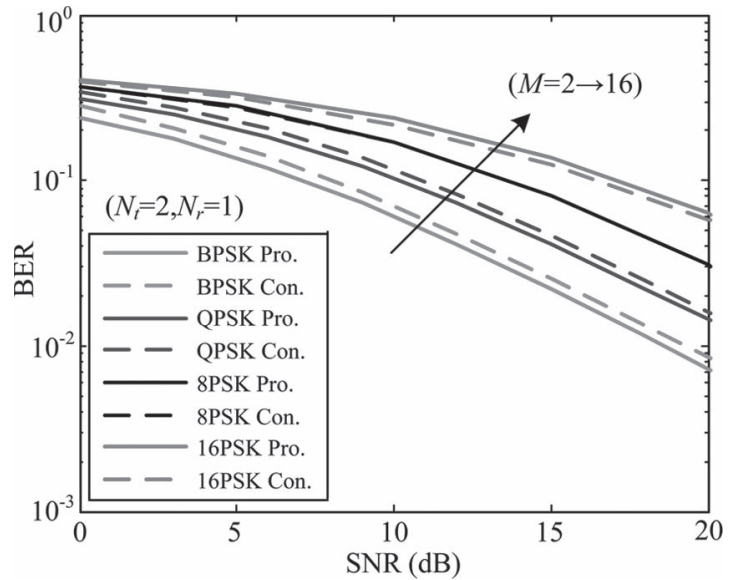

Fig. 4. BER performance of the proposed BTS-MAP and the conventional BTS-MAP schemes having $N_{t}=2, N_{r}=1$ and employing $M$-PSK signal sets.

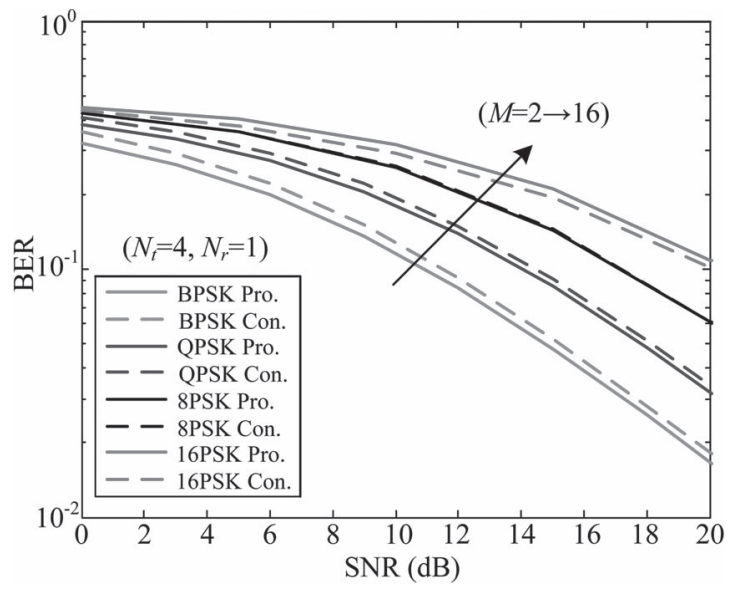

Fig. 5. BER performance of the proposed BTS-MAP and the conventiona BTS-MAP schemes associated with $N_{t}=4, N_{r}=1$ and $M$-PSK schemes.

377 conventional Gray mapping for classic 2-D constellations, the specific 378 SNR value only has a modest effect on the mapping gain of the 379 proposed scheme [9]. Observe in Fig. 4 that, for the case of $M>8$, the 380 conventional BTS-MAP outperforms the proposed BTS-MAP. This 381 result is consistent with the findings in Fig. 3, where the constraint of $382 P_{\text {Diff }}>P_{\text {Same }}$ is no longer met. Additionally, for the case of $M=8$, 383 it is found that the proposed BTS-MAP and the conventional BTS384 MAP achieve almost the same BER performance. This is due to the 385 fact that, for this scheme, we have $P_{\text {Diff }} \approx 0.5$. The aforementioned 386 trends of these BTS-MAP schemes recorded for SM are also visible in 387 Fig. 5, where $(4 \times 1)$-element SM systems are considered. Moreover, 388 in Fig. 6, the performance of the proposed BTS-MAP is investigated 389 in Nakagami- $m$ fading channels. As shown in Fig. 6, the proposed 390 scheme outperforms the conventional one in $(2 \times 1)$-element MIMO 391 channels having $m=1.5$ and $m=0.8$. Since we have a higher $P_{\text {Diff }}$ 392 for the case of $m=1.5$, the corresponding BER gain is more attractive 393 than that of $m=0.8 .^{1}$

\footnotetext{
${ }^{1}$ In our simulations, the value of $P_{\text {Diff }}$ for $m=1.5$ is approximately 0.7 , whereas this value for $m=0.8$ is about 0.55 . Moreover, our proposed BTSMAP can be also directly extended to the SM in conjunction with $M$-QAM modulation. Due to space limitations, the related simulation results are not included here.
}

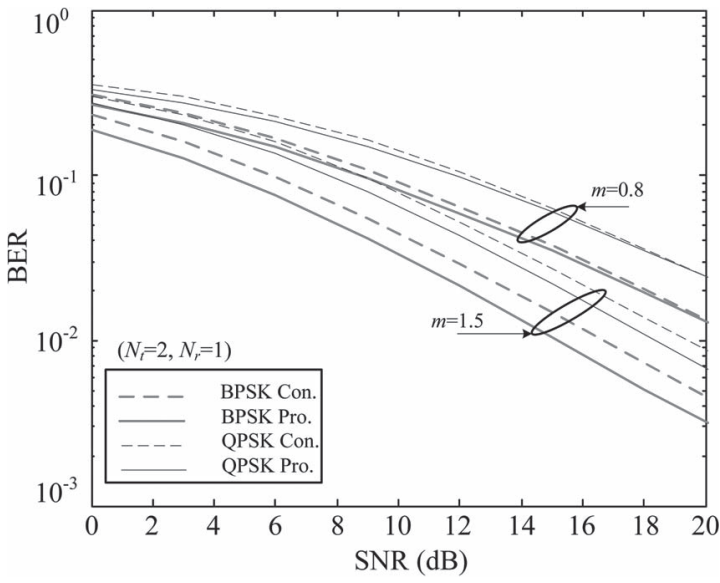

Fig. 6. BER performance of the proposed BTS-MAP and the conventional BTS-MAP schemes for $(2 \times 1)$-element Nakagami- $m$ channels.

\section{CONCLUSION}

A novel BTS-MAP scheme has been proposed for SM systems with 395 the objective of increasing the HDS and simultaneously reducing the 396 average HDD. Based on the theoretical analysis of the MED distribu- 397 tion of SM constellations, a criterion was proposed for the construction 398 of a beneficial BTS-MAP scheme for a specific MIMO setup. The 399 proposed mapping rule exhibited is attractive for employment in SM 400 systems. For achieving a further improved BER performance, our 401 further work will be focused on the integration of adaptive SM and 402 channel coding with the proposed scheme.

403

\section{REFERENCES}

404

[1] R. Mesleh, H. Haas, S. Sinanović, C. W. Ahn, and S. Yun, "Spatial 405 modulation," IEEE Trans. Veh. Technol., vol. 57, no. 4, pp. 2228-2241, 406 Jul. 2008.

407

[2] J. Jeganathan, A. Ghrayeb, L. Szczecinski, and A. Ceron, "Space shift 408 keying modulation for MIMO channels," IEEE Trans. Wireless Commun., 409 vol. 8, no. 7, pp. 3692-3703, Jul. 2009.

[3] S. Sugiura, S. Chen, and L. Hanzo, "A universal space-time architec- 411 ture for multiple-antenna aided systems," IEEE Commun. Surveys Tuts., 412 vol. 14, no. 2, pp. 401-420, 2nd Quart. 2012.

[4] M. Di Renzo, H. Haas, A. Ghrayeb, S. Sugiura, and L. Hanzo, "Spatial 414 modulation for generalized MIMO: Challenges, opportunities and imple- 415 mentation," Proc. IEEE, vol. 102, no. 1, pp. 56-103, Jan. 2014.

[5] M. Di Renzo, H. Haas, and P. M. Grant, "Spatial modulation for multiple- 417 antenna wireless systems: A survey," IEEE Commun. Mag., vol. 49, 418 no. 12, pp. 182-191, Dec. 2011.

[6] P. Yang, M. Di Renzo, Y. Xiao, S. Li, and L. Hanzo, "Design guidelines 420 for spatial modulation," IEEE Commun. Surveys Tuts., vol. 17, no. 1, 421 pp. 6-26, 1st Quart. 2015.

[7] L. Hanzo, S. X. Ng, T. Keller, and W. Webb, Quadrature Amplitude 423 Modulation: From Basics to Adaptive Trellis-Coded, Turbo-Equalised 424 and Space-Time Coded OFDM, CDMA and MC-CDMA Systems. 425 New York, NY, USA: Wiley, 2004

[8] L. Hanzo, T. H. Liew, and B. L. Yeap, Turbo Coding, Turbo Equali- 427 sation, and Space-Time Coding for Transmission over Fading Channels. 428 New York, NY, USA: Wiley-IEEE Press, 2002.

[9] E. Agreel, J. Lassing, E. G. Strom, and T. Ottosson, "Gray coding for 430 multilevel constellations in Gaussian noise," IEEE Trans. Inf. Theory, 431 vol. 53, no. 1, pp. 224-235, Jan. 2007.

[10] M. Di Renzo and H. Haas, "Bit error probability of spatial modula- 433 tion (SM-) MIMO over generalized fading channels," IEEE Trans. Veh. 434 Technol., vol. 61, no. 3, pp. 1124-1144, Mar. 2012.

[11] A. Younis, S. Sinanovic, M. Di Renzo, R. Y. Mesleh, and H. Haas, "Gen- 436 eralised sphere decoding for spatial modulation," IEEE Trans. Commun., 437 vol. 61, no. 7, pp. 2805-2815, Jul. 2013.

[12] M. Le, V. Ngo, H. Mai, X. Tran, and M. Di Renzo "Spatially modulated 439 orthogonal space-time block codes with non-vanishing determinants," 440 IEEE Trans. Commun., vol. 62, no. 1, pp. 85-98, Jan. 2014. 
442 [13] R. Y. Chang, S.-J. Lin, and W.-H. Chung, "Energy efficient transmis443 sion over space shift keying modulated MIMO channels," IEEE Trans. 444 Commun., vol. 60, no. 10, pp. 2950-2959, Oct. 2012.

445 [14] M. Di Renzo and H. Haas, "Improving the performance of space shift 446 keying (SSK) modulation via opportunistic power allocation," IEEE 447 Commun. Lett., vol. 14, no. 6, pp. 500-502, Jun. 2010.

448 [15] N. Serafimovski, M. Di Renzo, S. Sinanović, R. Y. Mesleh, and H. Haas, 449 "Fractional bit encoded spatial modulation (FBE-SM)," IEEE Commun. $450 \quad$ Lett., vol. 14, no. 5, pp. 429-431, May 2010.

451 [16] Y. Yang and S. Aïssa, "Bit-padding information guided channel hopping," 452 IEEE Commun. Lett., vol. 15, no. 2, pp. 163-165, Feb. 2011.

453 [17] R. Y. Mesleh, M. Di Renzo, H. Haas, and P. M. Grant, "Trellis coded 454 spatial modulation," IEEE Trans. Wireless Commun., vol. 9, no. 7, 455 pp. 2349-2361, Jul. 2010.

456 [18] E. Başar, Ü. Aygölü, E. Panayirci, and H. V. Poor, "New trellis code 457 design for spatial modulation," IEEE Trans. Wireless Commun., vol. 10, 458 no. 8, pp. 2670-2680, Aug. 2011.
[19] J. G. Proakis, Digital Communications, 3rd ed. Singapore: McGraw- 459 Hill, 1995.

460

[20] D. A. Freedman, Statistical Models: Theory and Practice. Cambridge, 461 U.K.: Cambridge Univ. Press, 2005.

[21] H. A. David and H. N. Nagaraja, Order Statistics, 3rd ed. New York, 463 NY, USA: Wiley, 2003.

[22] P. Yang, Y. Xiao, Y. Yi, and S. Li, "Adaptive spatial modulation for 465 wireless MIMO transmission systems," IEEE Commun. Lett., vol. 15, 466 no. 6, pp. 602-604, Jun. 2011.

467

[23] M. Maleki, H. R. Bahrami, S. Beygi, M. Kafashan, and N. H. Tran, 468 "Space modulation with CSI: Constellation design and performance eval- 469 uation," IEEE Trans. Veh. Technol., vol. 64, no. 4, pp. 1623-1634, 470 May 2013. 


\section{AUTHOR QUERIES}

\section{AUTHOR PLEASE ANSWER ALL QUERIES}

AQ1 = A citation for Table II was provided. The document, however, contains only one table. Please check. AQ2 = ML was expanded as "maximum likelihood". Please check if appropriate. Otherwise, please provide the corresponding expanded form.

AQ3 = The word "exhibit" in the sentence "The proposed mapping rule exhibits is attractive for employment in SM systems" was changed to "exhibited". Please check if appropriate. Otherwise, please make the necessary changes.

\section{END OF ALL QUERIES}

